\title{
Outlier Detection and Effects on Modeling
}

\author{
Christopher O. Arimie1, Emmanuel O. Biu², Maxwell A. Ijomah ${ }^{2}$ \\ ${ }^{1}$ Department of Radiology, University of Port Harcourt Teaching Hospital, Port Harcourt, Nigeria \\ ${ }^{2}$ Department of Mathematics and Statistics, University of Port Harcourt, Port Harcourt, Nigeria \\ Email: codarimie@yahoo.com, biu.emmanuel@uniport.edu.ng, Maxwell.ijomah@uniport.edu.ng
}

How to cite this paper: Arimie, C.O., Biu, E.O. and Ijomah, M.A. (2020) Outlier Detection and Effects on Modeling. Open Access Library Journal, 7: e6619. https://doi.org/10.4236/oalib.1106619

Received: July 16, 2020

Accepted: September 13, 2020

Published: September 16, 2020

Copyright $\odot 2020$ by author(s) and Open Access Library Inc.

This work is licensed under the Creative Commons Attribution International License (CC BY 4.0).

http://creativecommons.org/licenses/by/4.0/ (c) (i) Open Access

\begin{abstract}
In this work, a comprehensive framework for traditional outlier detection techniques based on simple and multiple linear regression models was studied. Two data sets were used for the illustration and evaluation of each class of outlier detection techniques (analytical and graphical methods). Outlier detection aims at identifying such outlier in order to improve the analytic of data and suitable model built. Furthermore, comparisons of the different methods were done to highlight the advantages, disadvantages and performance issues of each class of outlier detection techniques. The results show that by removing the influential points (or outliers), the model adequacy increased (from $\mathrm{R}^{2}=0.72$ to $\mathrm{R}^{2}=0.97$ ). It was observed that Jackknife residuals and Atkinson's measure methods are very useful in detecting outliers; hence, both methods were recommended for outliers' detection.
\end{abstract}

\section{Subject Areas}

Applied Statistical Mathematics

\section{Keywords}

Outliers' Detection, Classification and Comparisons, Simple and Multiple Linear Regression Models

\section{Background of the Study}

Outliers are observations that appear inconsistent with the remainder of the dataset. Bollen and Jackman [1] defined outliers as observations that are distinct from most of the data points in the sample. Hawkins [2] described outlier as an observation that deviates so much from other observations as to arouse suspicions that it was generated by different mechanisms. Dixon [3] sees outlier as values that are dubious in the eyes of the researcher and contaminants. Outliers are often present in real data but may go unnoticed because nowadays much da- 
ta are produced by computer without careful inspection or screening. Outliers may be mistakes in data entry or otherwise, accurate but unexpected observations which could shed new light on the phenomenon under study.

In general, outliers can be classified into two types: man-made one and random one. Man-made outliers may arise because of typographical error(s), misreporting of information, incorrect distribution assumption and sampling error. Random outliers, on the other hand, may arise because of random chance for drawing sample from a population. Presence of man-made or random outliers or both would seriously influence the result of statistical analysis including point and interval estimates and type 1 and type 11 errors. Outlier can cause us to misinterpret patterns in plots; it can affect visual resolution of remaining data in plot (forces observations into clusters) and may indicate that our model fails to capture important characteristics of the data. Unusual cases can substantially influence the fit of the ordinary least square model thus, leading to faulty conclusion. Some man-made outliers can be avoided by a strict data entry and rechecking process before conducting a statistical analysis. Data transformation is another way to reduce the influence of outliers (see Barnett and Lewis [4]; Montgomery [5]).

Quite a number of authors have proposed different methods of detecting outliers.

Abuzaid et al. [6], proposed a number of diagrammatical plots and hypothesis testing to detect outliers in the simple circular regression model. The work focused on detecting outlier in the down and Mardia's circular-circular regression model. Zhang et al. [7] presented a method which applies signal processing techniques to solve important problems in data mining. They introduced an outlier detection approach termed to find out, based on wavelet transform. The main idea in the method is to remove the clusters from the original data and then identify the outliers. Rousseeuw [8] proposed a depth based method to detect outlier. The data points are organized in layers in the data space according to the value of the point depth. Aggarwal and Yu [9] proposed a method which entails studying the projection from the rest of the data in a sparse data with high dimensionality. Arning et al. [10] introduced a method which relies on the observations such that after seeing a series of similar data, an element disturbing the series is considered an outlier.

Hodge and Austin [11] identified an efficient method for on-line classification and outlier detection in multivariate sensor data. This involved a comparative review to identify and distinguish their advantages and disadvantages and introducing a survey of contemporary outlier detection techniques. Sebert et al. [12] and Montgomery [5] asserts that to identify the existence of outliers the standardized residuals are computed and a large standardized residual of $(\mathrm{d}>3)$ indicates outlier. Worden et al. [13] used the concept of discordances to signal deviance from the norm.

Kitagawa [14] and Wing-Kam Fung and Bacon-Shone [15] applied Akaike information criterion (AIC) in detection of outliers by using (quasi) Bayesian 
approach with predictive likelihood. Belsey et al. [16] suggested standardizing each residual with an estimate of its standard deviation that is independent of the residual. This is accomplished by using as the estimate of variance for the $\mathrm{i}^{\text {th }}$ residual, the residual mean square from an analysis where that observation has been omitted. The result is a jackknife residual also called a fully studentized residual.

In this study, concentration is on the effect of outlier as well as detection methods on linear regression model. Specifically, we are concerned with observations that differ from the regression plane defined by the data. It is important to identify these types of outliers in regression modeling because the observations when undetected can lead to erroneous parameter estimates and inference from the model. Deleting outliers from a regression model can sometimes give completely different results as bias or distortion of estimates are removed. Identifying outliers in the real world data-base is important for improving the quality of original data and for reducing the impact of outliers. Identifying outliers and high-leverage points, is a fundamental step in the least-squares regression model building process. On this note, it is the purpose of this research to examine the effect of outliers on simple and multiple linear regression, compare different methods of detecting outlier and show its effect on modeling.

\section{Methods of Outlier Detection and Effects on Regression Model}

There are many methods for the detection of outliers in linear regression. They may be classified into graphical and analytical methods. The graphical methods include Scatter graph, Boxplot, Williams graph, Rankit graph (or Q-Q Plot) and graph of predicted residuals. The analytical methods are predicted residuals, standardized residuals, studentized residuals, Jack-knife residuals, Cook's distance, Different-in-fits (DFFITS) and Atkinson's measure.

The general linear model is given as

$$
y_{n \times 1}=X_{n \times k} \beta_{k \times 1}+\varepsilon_{n \times 1}
$$

where,

$y_{n \times 1}$ is the observation (dependent variables);

$X_{n \times k}$ is the design matrix including a constant;

$\varepsilon \sim N\left(0, \sigma^{2} I\right)$ is the error term;

$\beta=\left(\beta_{0}, \beta_{1}, \beta_{2}, \cdots, \beta_{k-1}\right)^{\prime}$ is the coefficient and $k$ is the number of covariates or predictors for each observation.

Then, the least square estimator is given as

$$
\beta=\left(X^{\prime} X\right)^{-1}\left(X^{\prime} Y\right)
$$

where the fitted (predicted) values for mean of $Y$ are

$$
\hat{Y}=\mathrm{X} \beta=\mathrm{X}\left(\mathrm{X}^{\prime} \mathrm{X}\right)^{-1}\left(\mathrm{X}^{\prime} Y\right)=\mathrm{H} Y
$$

where $H=X\left(X^{\prime} X\right)^{-1} X^{\prime}$ is the projection matrix (or hat matrix). 
The $I^{\text {th }}$ diagonal element of $H$ (given by $h_{i}=x_{i}^{\prime}\left(\mathrm{X}^{\prime} \mathrm{X}\right)^{-1} x_{i}$ is known as the leverage of the $t^{\text {th }}$ observation).

Similarly, the $i^{\text {th }}$ element of the residual vector

$$
\varepsilon_{i}=Y_{i}-\hat{Y}_{i}=(I-H) Y_{i}
$$

where $H$ is as defined in Equation (2.3), $I$ is the identity matrix and $Y_{i}$ is corresponding fitted value $(i=1,2, \cdots, n)$.

In this study, Outliers were detected using the following methods:

\subsection{Studentized and Standardized Residuals}

The Studentized residual is obtained by

$$
\varepsilon_{S . i}=\frac{\widehat{\varepsilon}_{i}}{\hat{\sigma} \sqrt{1-h_{i}}}=\frac{\widehat{\varepsilon}_{i}}{\sqrt{\hat{\sigma}^{2}\left(1-h_{i}\right)}}
$$

where $\hat{\sigma}$ is an appropriate estimate of $\sigma$, and the estimate of $\hat{\sigma}^{2}$ (Mean Residual Sum of Squares) is the internally studentized residuals. $h_{i}$ is leverage points as already defined.

$$
\hat{\sigma}^{2}=\frac{1}{n-k} \sum_{j=1}^{n} \hat{\varepsilon}_{j}
$$

where $k$ is the number of parameters in the model (Equation (2.1)).

Studentized residuals with large absolute values are considered large. If the regression model is appropriate, with no outlying observations, each Studentized residual follows a $t$ distribution with $n-k-1$ degrees of freedom.

CRITICAL: Each deleted residual has a student's t-distribution with $n-k-1$ degrees of freedom.

If the Studentized residual is divided by the estimates of its standard error so that the outcome is a residual with zero mean and standard deviation one, it becomes standardized residual denoted by

$$
\varepsilon_{S T . i}=\frac{\widehat{\varepsilon}_{i}}{s d(\sigma)}
$$

where $s d(\sigma)$ is the standard deviation of $\hat{\sigma}^{2}$ in Equation (2.6).

CRITICAL: The standardized residuals, $d_{i}>3$ potentially indicate outlier.

\subsection{Jackknife Residuals}

The jackknife residuals are residuals which with an assumption of normality of errors have a student distribution with $(n-k-1)$ degrees of freedom. The formula is:

$$
\varepsilon_{J . i}=\widehat{\varepsilon}_{S . i} \sqrt{\frac{(n-k-1)}{\left(n-k-\widehat{\varepsilon}_{S . i}^{2}\right)}}
$$

where $\varepsilon_{S . i}$ is the Studentized residuals Equation (2.5).

The jackknife residual examines the influence of individual point on the mean quadratic error of prediction. 
CRITICAL: Each deleted residual has a student's t-distribution with $n-k-1$ degrees of freedom.

\subsection{Predicted Residuals}

The predicted residual or Cross-validated residuals for observation $i$ is defined as the residual for the $i^{\text {th }}$ observation that results from dropping the $i^{\text {th }}$ observation from the parameter estimates. The sum of squares of predicted residual errors is called the PRESS statistic:

$$
\varepsilon_{P . i}=\frac{\varepsilon_{i}}{1-h_{i}}
$$

PRESS is called Prediction sum of squares; an assessment of your model's predictive ability. PRESS, similar to the residual sum of squares, is the sum of squares of the prediction error. In least squares regression, PRESS is calculated with the following formula:

$$
\operatorname{PRESS}=\sum_{i=1}^{n}\left(\frac{\varepsilon_{i}}{1-h_{i}}\right)^{2}
$$

where $\varepsilon_{i}=$ residual and $h_{i}=$ leverage value for the $i^{\text {th }}$ observation. In general, the smaller the PRESS value, the better the model's predictive ability.

\subsection{Cook's Distance}

Cook's distance $D_{i}$ of observation, $i$ is defined as the sum of all the changes in the regression model when observation $i$ is removed from it. Cook [17] proposed a statistic for detection of outlier, given as:

$$
D_{i}=\frac{\sum_{j=1}^{n}\left(y_{j}-\hat{y}_{j(i)}\right)^{2}}{k S^{2}}
$$

and $S^{2}=(n-k)^{-1} \varepsilon^{\prime} \varepsilon$ is the mean squared error of the regression model. Equivalently, it can be expressed using the leverage

$$
D_{i}=\frac{\varepsilon_{i}^{2}}{k S^{2}}\left[\frac{h_{i}}{\left(1-h_{i}\right)^{2}}\right]
$$

Here, $D_{i}$ measures the sum of squared changes in the predictions when observation " $i$ " is not used in estimating $\beta . D_{i}$ approximately follows $F(p, n-p)$ distribution.

CRITICAL: The cut off value of Cook-Statistic is $4 / n$.

\subsection{Difference-in-Fit (DFFIT)}

It is the difference between the predicted responses from the model constructed using complete data and the predicted responses from the model constructed by setting the $i^{\text {th }}$ observation aside. It is similar to cook's distance. Unlike cook's distance, it does not look at all of the predicted values with the $I^{\text {th }}$ observation set aside. It looks only at the predicted values for the $i^{\text {th }}$ observation. It combines le- 
verage and studentized residual (deleted $t$ residuals) values into one overall measure of how unusual an observation is. DFFIT is computed as follows:

$$
\text { DFFIT }=\varepsilon_{i}\left[\sqrt{\frac{n-k-1}{\sigma^{2}\left(1-h_{i}\right)-\varepsilon_{i}^{2}}}\right] \sqrt{\frac{h_{i}}{1-h_{i}}}
$$

where $\varepsilon_{i}=$ residual, $n=$ sample size, $k=$ the number of parameters in the model, $\sigma^{2}=$ variance and $h_{i}=$ leverage value for the $i^{\text {th }}$ observation.

CRITICAL: The cut off value of DFFIT is $2 \sqrt{\frac{k}{n}}$.

\subsection{Atkinson's Measure $\left(A_{i}\right)$}

It enhances the sensitivity of distance measures to high-leverage point. This modified version of cook's measure $D_{i}$ suggested by Atkinson is even more closely related to Belsey et al. (1980) [16] DFFITS and has the form

$$
A_{i}=\left|\varepsilon_{J . i}\right|\left[\sqrt{\frac{n-k}{k} \times \frac{h_{i}}{1-h_{i}}}\right]
$$

where $n, k, h_{i}$ are as defined in Equations (2.13) and $\varepsilon_{J . i}$ is the absolute value of Jackknife residuals.

This measure is also convenient for graphical interpretation.

\subsection{Scatter Graph and Box Plot}

Scatter plot is a line of best fit (alternatively called "trendline") drawn in order to study the relationship between the variables measured. For a set of data variables (dimensions) $X_{1}, X_{2}, \cdots, X_{k}$, the scatter plot matrix shows all the pairwise scatter plots of the variables on the dependent variable.

A box plot is a method for graphically depicting groups of numerical data through their quartiles (i.e. Mean, Median Mode, quartiles). Box plots may also have lines extending vertically from the boxes (whiskers) indicating variability outside the upper and lower quartiles. It is also called box-and-whisker plot and box-and-whisker diagram. Outliers may be plotted as individual points and it can be used for outlier detection in regression model, where the primary aim here is not to fit a regression model but find out outliers using regression and to improve a regression model by removing the outliers.

\subsection{Willams Graph, Rankit Graph (or Q-Q Plot) and Predicted Residuals Graph}

The Williams graph (Williams [18]) has the diagonal elements $H_{i i}$ on the $x$-axis and the jackknife residuals $\hat{\varepsilon}_{J i}$ on the $y$-axis. Two boundary lines are drawn, the first for outlier $y=t_{0.95}(n-k-i)$, and the second for high leverages, $x=$ $2 k / n$. Note: $t_{0.95}(n-k-1)$ is the $95 \%$ quantile of the student distribution with ( $n$ $-k-1)$ degrees of freedom.

The Q-Q plot (or Rankit Graph) has the quantile of the standardized normal distribution $\mu p_{i}$ for $P_{i}=i /(n+1)$ on the $x$-axis and the ordered residuals 
$\hat{\varepsilon}_{S . i}, \hat{\varepsilon}_{P . i}, \hat{\varepsilon}_{J . i}$ i.e. increasingly ordered values of various types of residuals on the $\mathrm{y}$-axis.

The graph of predicted residuals (or Predicted Residuals Graph) has the predicted residuals $\hat{\varepsilon}_{P i}$ on the $x$-axis and the ordinary residuals $\hat{\varepsilon}_{i}$ (Equation (3.4)) on the $y$-axis. The outlier can easily be detected by their location, as they lie outside the line $y=x$ far from its central pattern (Meloun and Militky [19]).

\section{Methodology}

Two sets of data (see Appendix), were collected and used to build the regression models. The first was data of rainfall (in Millimetres) and yield of Wheat (in kg) and the second was data of agricultural products (Crop production, Livestock, Forestry and fishing) and Nigeria gross domestic product (GDP). The seven analytical and five graphical methods listed above were then applied to detect outliers in the simple and multiple linear regression models.

\section{Results and Discussion}

\subsection{Simple Linear Regression}

Simple linear regression of rainfall (Millimetres) on yield of Wheat (kg) was done, using Minitab 17 software to obtain the residuals of the model for outlier detection Methods to be applied.

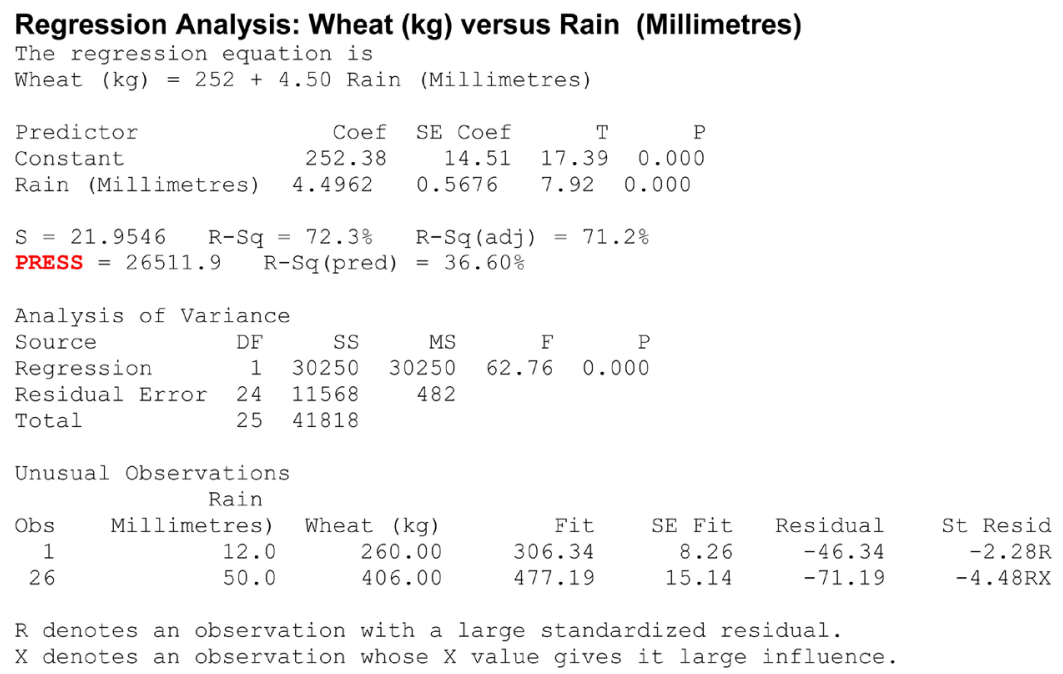

\subsubsection{Outlier Detection Methods}

The seven analytical and five graphical methods for outlier detection discussed in Section 2 were used to detect outliers when the regression model was built using the rainfall and yield of wheat data. The results of the computation using the analytical methods are shown in Table 1 while Table 2 gives a summary of the number of outliers detected by each analytical method. Figures 1(a)-(f) show the scatter plot of outliers detected by the methods indicated. Figures 2(a)-(f) show the box plot of outliers detected by the methods indicated while Figures 3(a)-(d) show the Rankit Graph (or Q-Q Plot) of outliers detected. 


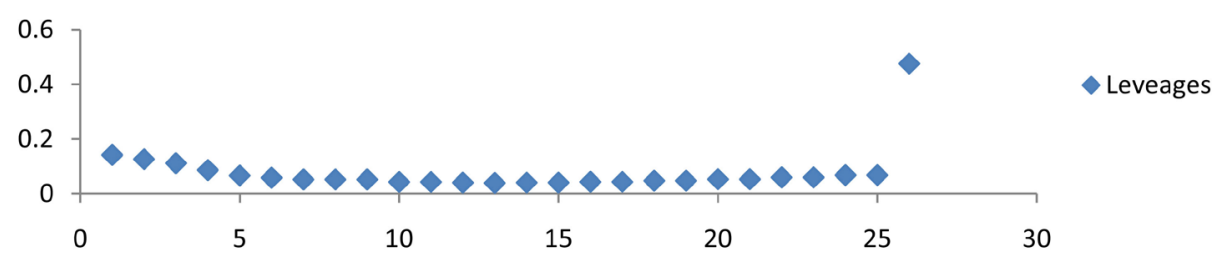

(a)

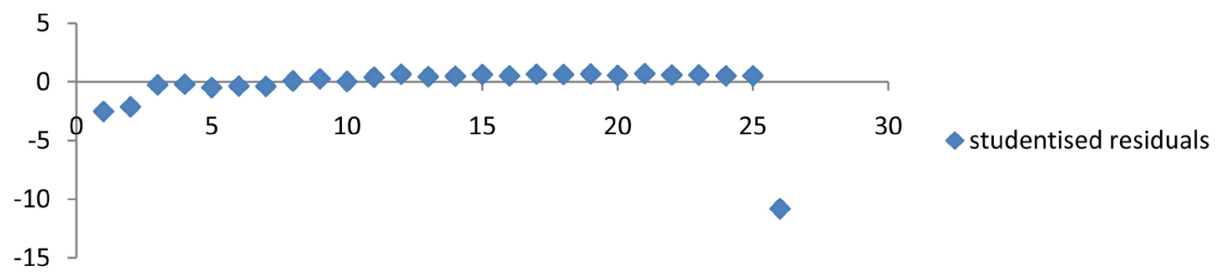

(b)

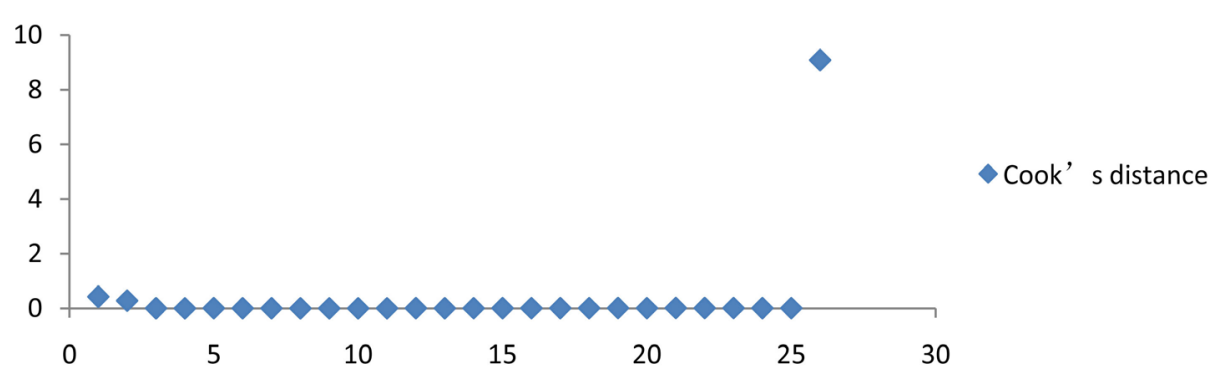

(c)

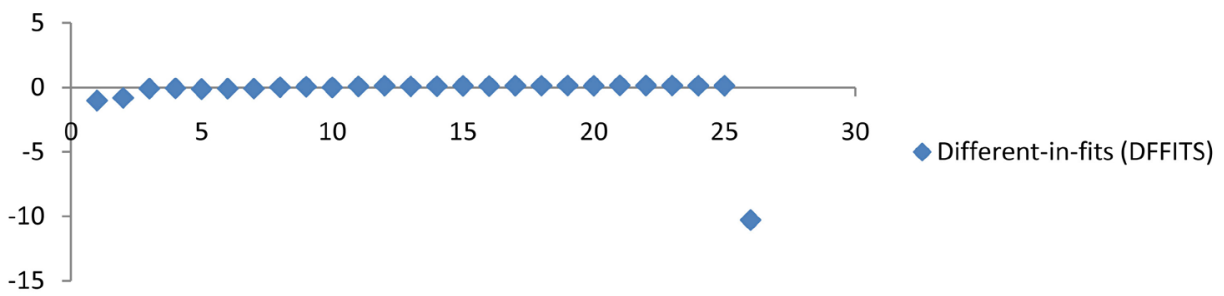

(d)

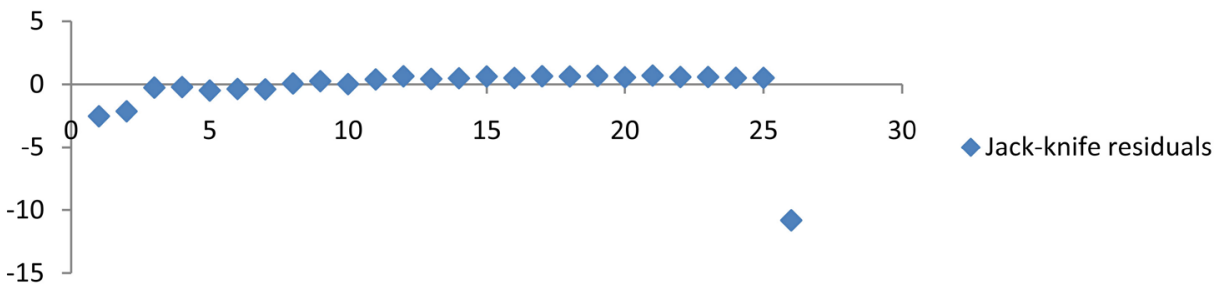

(e)

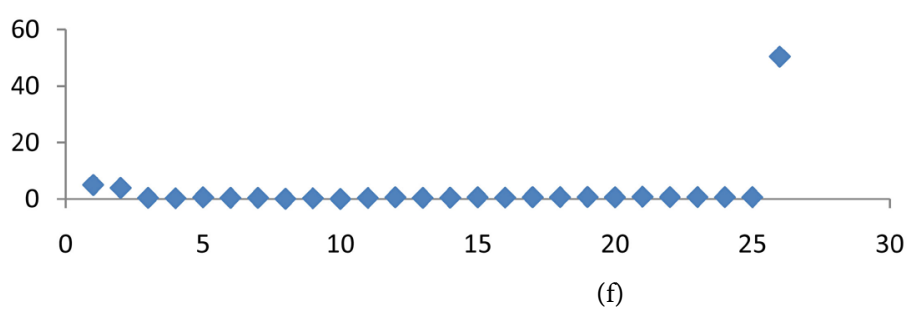

$\diamond$ Atkinson' s measure.

Figure 1. Scatter plots showing outliers detected by each of the analytical methods indicated. (a) Leveages; (b) Studentised residuals; (c) Cook's distance; (d) Different-in-fits (DFFITS); (e) Jack-knife residuals; (f) Atkinson's measure. 


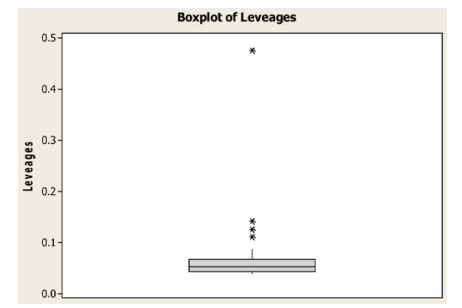

(a)

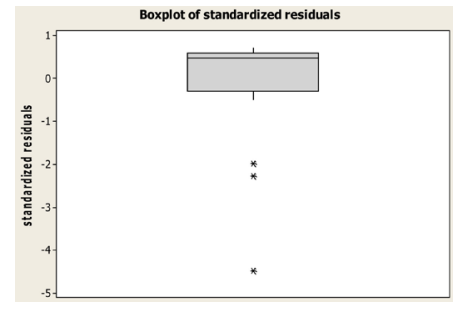

(c)

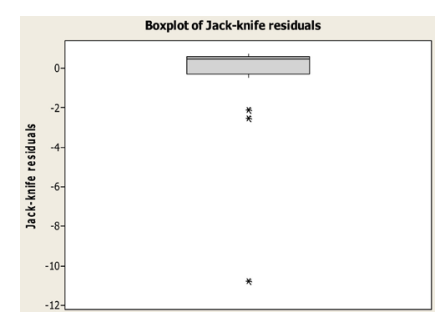

(e)

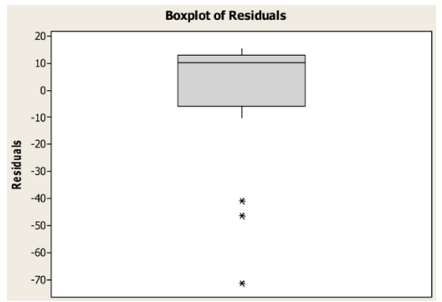

(b)

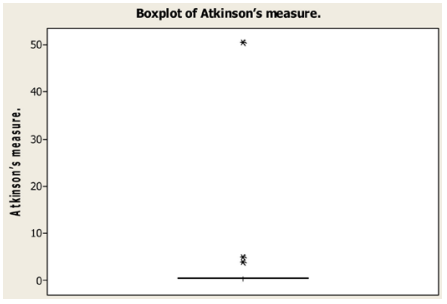

(d)

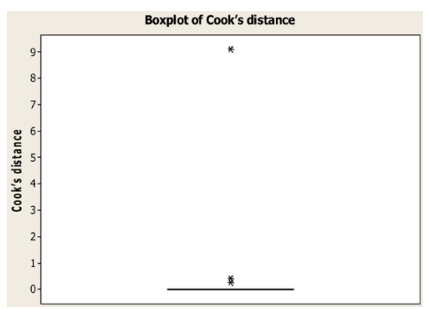

(f)

Figure 2. Boxplots showing outliers detected by each of the analytical methods indicated. (a) Leverages; (b) Residuals; (c) Standardized residuals; (d) Atkinson's measure; (e) Jack-knife residual; (f) Cook's distance.

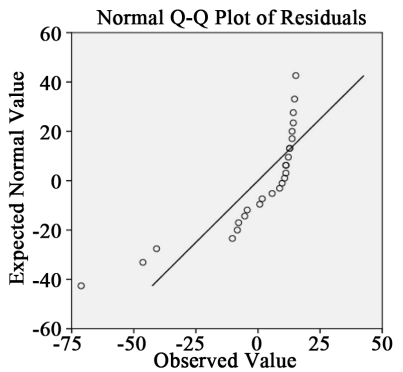

(a)

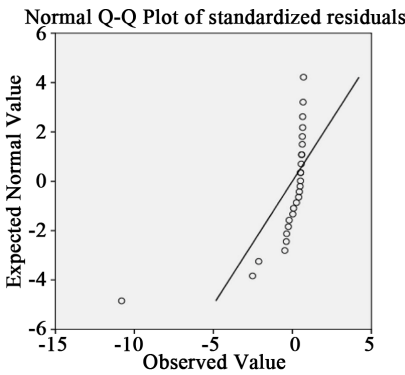

(c)

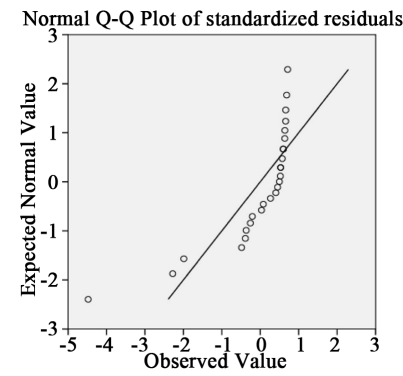

(b)

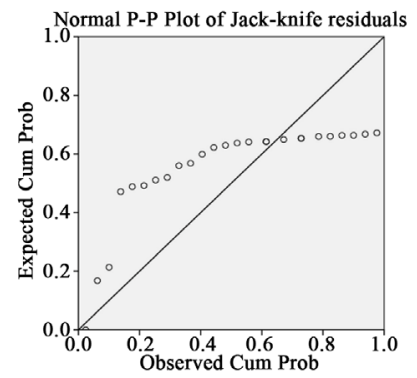

(d)

Figure 3. Rankit graph (or Q-Q Plot) of outliers detected by each of the analytical methods indicated. (a) Q-Q plot of Residuals; (b) Standardized Residuals; (c) Studentized Residuals; (d) Jack-knife Residuals. 
Table 1. Outliers detected using the analytical methods.

\begin{tabular}{|c|c|c|c|c|c|c|c|c|}
\hline $\begin{array}{c}\text { Leverages } \\
h_{i}\end{array}$ & $\begin{array}{c}\text { Residuals } \\
\varepsilon_{i}\end{array}$ & $\begin{array}{c}\text { Standardized } \\
\text { residuals } \\
\varepsilon_{S T . i}\end{array}$ & $\begin{array}{c}\text { Studentised } \\
\text { residuals } \\
\varepsilon_{S . i}\end{array}$ & $\begin{array}{l}\text { Predicted } \\
\text { residuals } \\
\varepsilon_{P . i}\end{array}$ & $\begin{array}{c}\text { Cook's } \\
\text { distance } \\
D_{i}\end{array}$ & $\begin{array}{c}\text { Different-in-fits } \\
\text { (DFFITS) } \\
\text { DFFit }\end{array}$ & $\begin{array}{c}\text { Jack-knife } \\
\text { residuals } \\
\varepsilon_{J . i}\end{array}$ & $\begin{array}{c}\text { Atkinson's } \\
\text { measure } \\
A_{i}\end{array}$ \\
\hline 0.141601 & -46.3356 & -2.27796 & -2.5189 & -53.979094 & 0.42799 & -1.023 & -2.518867 & 5.0118657 \\
\hline 0.125665 & -40.8318 & -1.989 & -2.1306 & -46.700407 & 0.2843 & -0.8077 & -2.130629 & 3.9571423 \\
\hline 0.111065 & -5.328 & -0.2574 & -0.2523 & -5.9936891 & 0.00414 & -0.0892 & -0.252329 & 0.4369446 \\
\hline 0.085876 & -4.3204 & -0.20582 & -0.2017 & -4.7262735 & 0.00199 & -0.0618 & -0.201665 & 0.3028088 \\
\hline 0.066033 & -10.3128 & -0.48606 & -0.4782 & -11.041932 & 0.00835 & -0.1271 & -0.478185 & 0.6228977 \\
\hline 0.058116 & -7.809 & -0.3665 & -0.3598 & -8.2908299 & 0.00414 & -0.0894 & -0.359792 & 0.4378303 \\
\hline 0.051536 & -8.3052 & -0.38843 & -0.3815 & -8.7564736 & 0.0041 & -0.0889 & -0.381453 & 0.4356032 \\
\hline 0.051536 & 1.6948 & 0.07926 & 0.0776 & 1.78688912 & 0.00017 & 0.0181 & 0.077601 & 0.0886176 \\
\hline 0.051536 & 5.6948 & 0.26634 & 0.2611 & 6.00423421 & 0.00193 & 0.0609 & 0.261118 & 0.2981865 \\
\hline 0.042385 & 0.7024 & 0.03269 & 0.032 & 0.73348893 & 0.00002 & 0.0067 & 0.032002 & 0.0329837 \\
\hline 0.042385 & 8.7024 & 0.40506 & 0.3979 & 9.08757695 & 0.00363 & 0.0837 & 0.397894 & 0.4100941 \\
\hline 0.039815 & 14.2061 & 0.66035 & 0.6524 & 14.7951697 & 0.00904 & 0.1328 & 0.6524 & 0.6508272 \\
\hline 0.038581 & 9.7099 & 0.45106 & 0.4434 & 10.0995508 & 0.00408 & 0.0888 & 0.443447 & 0.4351884 \\
\hline 0.040123 & 10.7175 & 0.49827 & 0.4903 & 11.1654931 & 0.00519 & 0.1002 & 0.490322 & 0.4911064 \\
\hline 0.040123 & 13.7175 & 0.63774 & 0.6297 & 14.2908935 & 0.0085 & 0.1287 & 0.62967 & 0.6306783 \\
\hline 0.042899 & 11.2213 & 0.52244 & 0.5144 & 11.724259 & 0.00612 & 0.1089 & 0.514373 & 0.533493 \\
\hline 0.042899 & 14.2213 & 0.66212 & 0.6542 & 14.8587244 & 0.00983 & 0.1385 & 0.654182 & 0.678498 \\
\hline 0.047012 & 13.7251 & 0.64039 & 0.6323 & 14.4021751 & 0.01012 & 0.1404 & 0.632332 & 0.6880368 \\
\hline 0.047012 & 14.7251 & 0.68705 & 0.6793 & 15.4515062 & 0.01164 & 0.1509 & 0.679298 & 0.7391394 \\
\hline 0.052461 & 12.2289 & 0.57222 & 0.564 & 12.9059595 & 0.00906 & 0.1327 & 0.564033 & 0.6501741 \\
\hline 0.052461 & 15.2289 & 0.7126 & 0.7051 & 16.0720561 & 0.01406 & 0.1659 & 0.705095 & 0.8127804 \\
\hline 0.059247 & 12.7327 & 0.59794 & 0.5898 & 13.5345835 & 0.01126 & 0.148 & 0.58976 & 0.7250636 \\
\hline 0.059247 & 12.7327 & 0.59794 & 0.5898 & 13.5345835 & 0.01126 & 0.148 & 0.58976 & 0.7250636 \\
\hline 0.067369 & 11.2365 & 0.52997 & 0.5219 & 12.0481734 & 0.01014 & 0.1403 & 0.521874 & 0.6871421 \\
\hline 0.067369 & 11.2365 & 0.52997 & 0.5219 & 12.0481734 & 0.01014 & 0.1403 & 0.521874 & 0.6871421 \\
\hline 0.475646 & -71.1915 & -4.47807 & -10.8101 & -135.76992 & 9.09517 & -10.2957 & -10.81004 & 50.438549 \\
\hline
\end{tabular}

Footnote: $d_{i}>3 ; t_{0.05}(22)=2.074 ; 4 / n=4 / 25=0.160 ; 2 \sqrt{\frac{k}{n}}=0.566 ; t_{0.05}(22)=2.074 A_{i}>3$.

Table 2. Summary of the results in Table 1.

\begin{tabular}{|c|c|c|c|c|c|c|}
\hline Outlier detection methods & $\begin{array}{c}\text { Standardized } \\
\text { residuals } \\
\varepsilon_{S T . i}\end{array}$ & $\begin{array}{c}\text { Studentised } \\
\text { residuals } \\
\varepsilon_{S . i}\end{array}$ & $\begin{array}{c}\text { Cook's } \\
\text { distance } \\
D_{i}\end{array}$ & $\begin{array}{c}\text { Different-in-fits } \\
\text { (DFFITS) } \\
\text { DFFit }\end{array}$ & $\begin{array}{c}\text { Jack-knife } \\
\text { residuals } \\
\varepsilon_{J . i}\end{array}$ & $\begin{array}{c}\text { Atkinson's } \\
\text { measure } \\
A_{i}\end{array}$ \\
\hline Numbers of outliers & 1 & 3 & 3 & 3 & 3 & 3 \\
\hline
\end{tabular}




\subsubsection{Simple Linear Regression without Outliers (Using Mean} Imputation Methods)

Similarly, Simple linear regression of rainfall (Millimetres) on Wheat $(\mathrm{kg})$ was done without the outliers identified (Mean imputation methods). The results of the computation are shown in Table 3 and summary of the number of outliers detected is shown in Table 4.

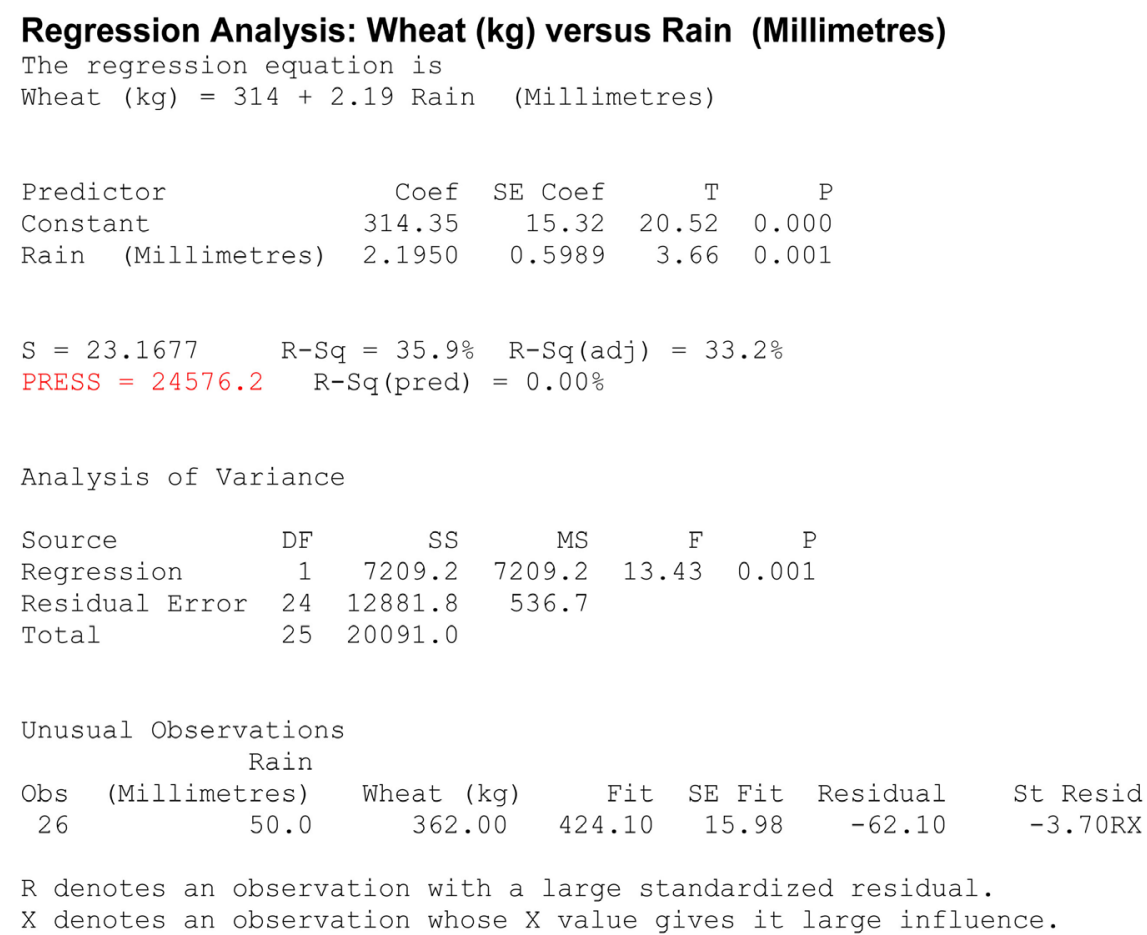

\subsubsection{Simple Linear Regression without Outliers (Using Remove Methods]}

Also, Simple linear regression of rainfall (Millimetres) on Wheat $(\mathrm{kg})$ done without outliers identified (remove Methods), has results of the computation displayed in Table 5 and summary of the number of outliers detected is shown in Table 6.

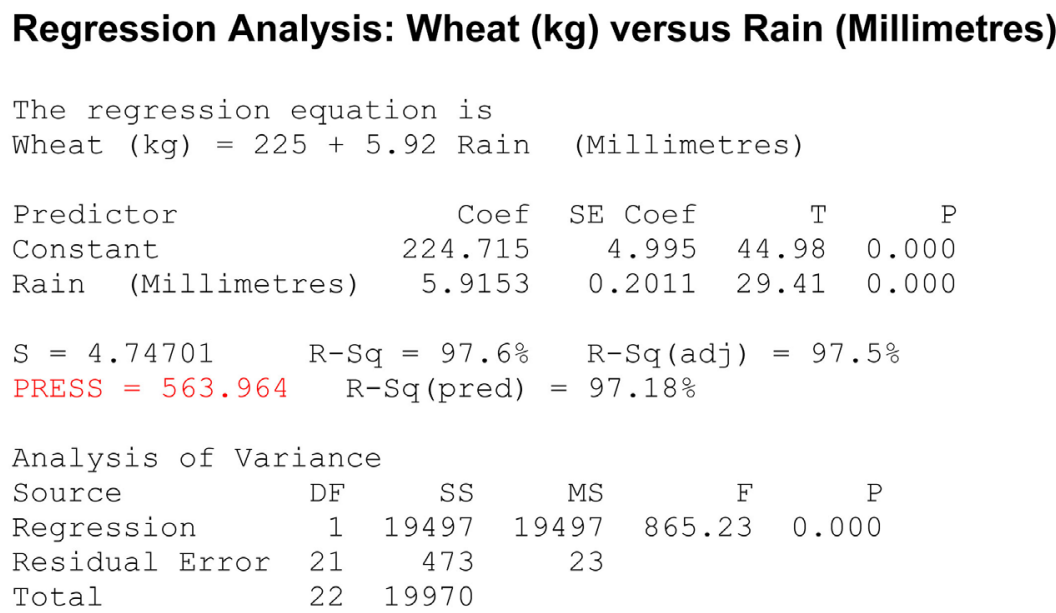


Table 3. Outliers detected using the analytical methods when the regression model was considered without outlier (Mean imputation method).

\begin{tabular}{|c|c|c|c|c|c|c|c|c|}
\hline $\begin{array}{c}\text { Leverages } \\
h_{i}\end{array}$ & $\begin{array}{c}\text { Residuals } \\
\varepsilon_{i}\end{array}$ & $\begin{array}{c}\text { Standardized } \\
\text { residuals } \\
\varepsilon_{S T . i}\end{array}$ & $\begin{array}{c}\text { Studentised } \\
\text { residuals } \\
\varepsilon_{S . i}\end{array}$ & $\begin{array}{c}\text { Predicted } \\
\text { residuals } \\
\varepsilon_{P . i}\end{array}$ & $\begin{array}{c}\text { Cook's } \\
\text { distance } \\
D_{i}\end{array}$ & $\begin{array}{c}\text { Different-in-fits } \\
\text { (DFFITS) } \\
\text { DFFit }\end{array}$ & $\begin{array}{c}\text { Jack-knife } \\
\text { residuals } \\
\varepsilon_{J . i}\end{array}$ & $\begin{array}{c}\text { Atkinson's } \\
\text { measure } \\
A_{i}\end{array}$ \\
\hline 0.141601 & 21.3066 & 0.99263 & 0.99232 & 24.82132 & 0.08127 & 0.40303 & 0.992313 & 1.974436 \\
\hline 0.125665 & 19.1117 & 0.88222 & 0.878 & 21.85856 & 0.05593 & 0.33286 & 0.877999 & 1.630676 \\
\hline 0.111065 & -35.0833 & -1.60614 & -1.66431 & -39.4667 & 0.16116 & -0.58829 & -1.66431 & 2.881998 \\
\hline 0.085876 & -29.4732 & -1.33058 & -1.35345 & -32.242 & 0.08316 & -0.41483 & -1.35344 & 2.032256 \\
\hline 0.066033 & -30.8631 & -1.37845 & -1.40624 & -33.0452 & 0.06717 & -0.37392 & -1.40624 & 1.83181 \\
\hline 0.058116 & -26.0581 & -1.15894 & -1.16768 & -27.6659 & 0.04144 & -0.29005 & -1.16768 & 1.420953 \\
\hline 0.051536 & -24.2531 & -1.07491 & -1.07856 & -25.5709 & 0.03139 & -0.25141 & -1.07856 & 1.231672 \\
\hline 0.051536 & -14.2531 & -0.63171 & -0.62361 & -15.0276 & 0.01084 & -0.14536 & -0.62362 & 0.712144 \\
\hline 0.051536 & -10.2531 & -0.45442 & -0.44678 & -10.8102 & 0.00561 & -0.10415 & -0.44678 & 0.510203 \\
\hline 0.042385 & -10.643 & -0.46945 & -0.46169 & -11.1141 & 0.00488 & -0.09713 & -0.46169 & 0.475847 \\
\hline 0.042385 & -2.643 & -0.11658 & -0.11416 & -2.75998 & 0.0003 & -0.02402 & -0.11416 & 0.117658 \\
\hline 0.039815 & 5.1621 & 0.22739 & 0.22284 & 5.376151 & 0.00107 & 0.04538 & 0.222842 & 0.222305 \\
\hline 0.038581 & 2.9671 & 0.13062 & 0.12791 & 3.086167 & 0.00034 & 0.02562 & 0.127915 & 0.125533 \\
\hline 0.040123 & 8.5772 & 0.37788 & 0.37103 & 8.935728 & 0.00298 & 0.07586 & 0.371029 & 0.371623 \\
\hline 0.040123 & 11.5772 & 0.51005 & 0.50204 & 12.06113 & 0.00544 & 0.10264 & 0.502039 & 0.502843 \\
\hline 0.042899 & 11.3822 & 0.50219 & 0.49422 & 11.89237 & 0.00565 & 0.10463 & 0.49422 & 0.512591 \\
\hline 0.042899 & 14.3822 & 0.63455 & 0.62647 & 15.02684 & 0.00902 & 0.13263 & 0.626467 & 0.649753 \\
\hline 0.047012 & 16.1873 & 0.71573 & 0.70826 & 16.98584 & 0.01264 & 0.15731 & 0.70826 & 0.770653 \\
\hline 0.047012 & 17.1873 & 0.75994 & 0.75306 & 18.03517 & 0.01424 & 0.16726 & 0.753055 & 0.819394 \\
\hline 0.052461 & 16.9923 & 0.75348 & 0.7465 & 17.93309 & 0.01572 & 0.17565 & 0.746498 & 0.860506 \\
\hline 0.052461 & 19.9923 & 0.88651 & 0.88241 & 21.09918 & 0.02176 & 0.20763 & 0.882412 & 1.017178 \\
\hline 0.059247 & 19.7973 & 0.88102 & 0.87677 & 21.0441 & 0.02444 & 0.22003 & 0.876765 & 1.077914 \\
\hline 0.059247 & 19.7973 & 0.88102 & 0.87677 & 21.0441 & 0.02444 & 0.22003 & 0.876765 & 1.077914 \\
\hline 0.067369 & 20.6024 & 0.92083 & 0.9178 & 22.09062 & 0.03063 & 0.24667 & 0.917801 & 1.208451 \\
\hline 0.067369 & 20.6024 & 0.92083 & 0.9178 & 22.09062 & 0.03063 & 0.24667 & 0.917801 & 1.208451 \\
\hline 0.475646 & -62.1019 & -3.70178 & -5.53251 & -118.435 & 6.21512 & -5.26929 & -5.53252 & 25.81417 \\
\hline
\end{tabular}

Footnote: $d_{i}>3 ; t_{0.05}(22)=2.074 ; 4 / n=4 / 25=0.160 ; 2 \sqrt{\frac{k}{n}}=0.566 ; t_{0.05}(22)=2.074 A_{i}>3$.

Table 4. Summary of the results in Table 3.

\begin{tabular}{ccccccc}
\hline Outlier detection methods & $\begin{array}{c}\text { Standardized } \\
\text { residuals } \\
\varepsilon_{S T . i}\end{array}$ & $\begin{array}{c}\text { Studentised } \\
\text { residuals }\end{array}$ & $\begin{array}{c}\text { Cook's } \\
\text { distance } \\
\varepsilon_{S . i}\end{array}$ & $\begin{array}{c}\text { Different-in-fits } \\
\text { (DFFITS) } \\
\text { DFFit }\end{array}$ & $\begin{array}{c}\text { Jack-knife } \\
\text { residuals } \\
\varepsilon_{J . i}\end{array}$ & $\begin{array}{c}\text { Atkinson's } \\
\text { measure } \\
A_{i}\end{array}$ \\
\hline Numbers of outliers & 1 & 1 & 1 & 1 & 1 & 1 \\
\hline
\end{tabular}


Table 5. Outliers detected using the analytical methods when the regression model was considered without outlier (Remove method).

\begin{tabular}{|c|c|c|c|c|c|c|c|c|}
\hline $\begin{array}{c}\text { Leverages } \\
h_{i}\end{array}$ & $\begin{array}{c}\text { Residuals } \\
\varepsilon_{i}\end{array}$ & $\begin{array}{c}\text { Standardized } \\
\text { residuals } \\
\varepsilon_{S T . i}\end{array}$ & $\begin{array}{c}\text { Studentised } \\
\text { residuals } \\
\varepsilon_{S . i}\end{array}$ & $\begin{array}{l}\text { Predicted } \\
\text { residuals } \\
\quad \varepsilon_{P . i}\end{array}$ & $\begin{array}{c}\text { Cook's } \\
\text { distance } \\
D_{i}\end{array}$ & $\begin{array}{c}\text { Different-in-fits } \\
\text { (DFFITS) } \\
\text { DFFit }\end{array}$ & $\begin{array}{c}\text { Jack-knife } \\
\text { residuals } \\
\varepsilon_{J . i}\end{array}$ & $\begin{array}{c}\text { Atkinson's } \\
\text { measure } \\
A_{i}\end{array}$ \\
\hline 0.235643 & 2.47097 & 0.59539 & 0.58601 & 3.232743 & 0.054642 & 0.325373 & 0.586445 & 1.527277 \\
\hline 0.168539 & 0.64045 & 0.14796 & 0.14447 & 0.770271 & 0.002219 & 0.065044 & 0.14463 & 0.305421 \\
\hline 0.115793 & -8.19007 & -1.83481 & -1.95405 & -9.26262 & 0.220434 & -0.70713 & -1.94784 & 3.306202 \\
\hline 0.094803 & -7.10534 & -1.57323 & -1.63467 & -7.8495 & 0.12961 & -0.52902 & -1.63157 & 2.476611 \\
\hline 0.077403 & -9.0206 & -1.97838 & -2.14044 & -9.7774 & 0.164186 & -0.61998 & -2.13181 & 2.896224 \\
\hline 0.077403 & 0.9794 & 0.2148 & 0.20985 & 1.061569 & 0.001935 & 0.060784 & 0.210082 & 0.285412 \\
\hline 0.077403 & 4.9794 & 1.09207 & 1.09737 & 5.397156 & 0.050029 & 0.317853 & 1.097113 & 1.490512 \\
\hline 0.053371 & -2.85112 & -0.61731 & -0.60798 & -3.01187 & 0.010743 & -0.14436 & -0.60841 & 0.677595 \\
\hline 0.053371 & 5.14888 & 1.11481 & 1.12164 & 5.439174 & 0.035035 & 0.266328 & 1.121311 & 1.248821 \\
\hline 0.046738 & 9.23361 & 1.99226 & 2.15894 & 9.68633 & 0.097302 & 0.478049 & 2.150042 & 2.232992 \\
\hline 0.043695 & 3.31835 & 0.71483 & 0.70625 & 3.46997 & 0.011674 & 0.150966 & 0.70665 & 0.708489 \\
\hline 0.048377 & 1.48783 & 0.32129 & 0.31432 & 1.563466 & 0.002624 & 0.07087 & 0.314642 & 0.332748 \\
\hline 0.048377 & 4.48783 & 0.96913 & 0.96766 & 4.715975 & 0.023873 & 0.218178 & 0.96773 & 1.023417 \\
\hline 0.056102 & 0.57257 & 0.12415 & 0.1212 & 0.606602 & 0.000458 & 0.029548 & 0.121338 & 0.138751 \\
\hline 0.056102 & 3.57257 & 0.77464 & 0.767 & 3.784911 & 0.017833 & 0.186992 & 0.767367 & 0.877488 \\
\hline 0.067416 & 1.6573 & 0.36152 & 0.35391 & 1.777105 & 0.004724 & 0.095156 & 0.354262 & 0.446759 \\
\hline 0.067416 & 2.6573 & 0.57966 & 0.57028 & 2.849395 & 0.012145 & 0.153328 & 0.570708 & 0.719718 \\
\hline 0.082319 & -1.25796 & -0.27663 & -0.27046 & -1.3708 & 0.003432 & -0.081 & -0.27074 & 0.380338 \\
\hline 0.082319 & 1.74204 & 0.38308 & 0.37516 & 1.898307 & 0.006582 & 0.112363 & 0.375527 & 0.527541 \\
\hline 0.100811 & -2.17322 & -0.48279 & -0.47379 & -2.41687 & 0.013066 & -0.15864 & -0.47421 & 0.744748 \\
\hline 0.100811 & -2.17322 & -0.48279 & -0.47379 & -2.41687 & 0.013066 & -0.15864 & -0.47421 & 0.744748 \\
\hline 0.122893 & -5.08848 & -1.14457 & -1.15354 & -5.80144 & 0.091776 & -0.43179 & -1.15311 & 2.024514 \\
\hline 0.122893 & -5.08848 & -1.14457 & -1.15354 & -5.80144 & 0.091776 & -0.43179 & -1.15311 & 2.024514 \\
\hline 0.067369 & 11.2365 & 0.52997 & 0.5219 & 12.04817 & 0.01014 & 0.1403 & 0.521122 & 0.656941 \\
\hline
\end{tabular}

Footnote: $d_{i}>3 ; t_{0.05}(22)=2.074 ; 4 / n=4 / 25=0.160 ; 2 \sqrt{\frac{k}{n}}=0.566 ; t_{0.05}(22)=2.074 A_{i}>3$.

Table 6. Summary of results in Table 5.

\begin{tabular}{ccccccc}
\hline & $\begin{array}{c}\text { Standardized } \\
\text { residuals } \\
\varepsilon_{S T . i}\end{array}$ & $\begin{array}{c}\text { Studentised } \\
\text { residuals }\end{array}$ & $\begin{array}{c}\text { Cook's } \\
\text { distance } \\
\varepsilon_{S . i}\end{array}$ & $\begin{array}{c}\text { Different-in-fits } \\
\text { (DFFITS) }\end{array}$ & $\begin{array}{c}\text { Jack-knife } \\
\text { residuals } \\
\varepsilon_{J . i}\end{array}$ & $\begin{array}{c}\text { Atkinson's } \\
\text { measure } \\
A_{i}\end{array}$ \\
\hline Numbers of outliers & 0 & 0 & 0 & 0 & 1 & 1 \\
\hline
\end{tabular}


Table 7. Outliers detected using the analytical methods.

\begin{tabular}{|c|c|c|c|c|c|c|c|c|}
\hline $\begin{array}{c}\text { Leverages } \\
h_{i}\end{array}$ & $\begin{array}{l}\text { Residuals } \\
\varepsilon_{i}\end{array}$ & $\begin{array}{c}\text { Standardized } \\
\text { residuals } \\
\varepsilon_{S T . i}\end{array}$ & $\begin{array}{c}\text { Studentised } \\
\text { residuals } \\
\varepsilon_{S . i}\end{array}$ & $\begin{array}{l}\text { Predicted } \\
\text { residuals } \\
\quad \varepsilon_{P . i}\end{array}$ & $\begin{array}{c}\text { Cook's } \\
\text { distance } \\
D_{i}\end{array}$ & $\begin{array}{c}\text { Different-in-fits } \\
\text { (DFFITS) } \\
\text { DFFit }\end{array}$ & $\begin{array}{c}\text { Jack-knife } \\
\text { residuals } \\
\varepsilon_{J . i}\end{array}$ & $\begin{array}{c}\text { Atkinson's } \\
\text { measure } \\
A_{i}\end{array}$ \\
\hline 0.006745 & 40,628 & 0.28615 & 0.28546 & $40,903.9$ & 0.000111 & 0.02352 & 0.864667 & 0.997557 \\
\hline 0.006745 & 40,792 & 0.2873 & 0.28661 & $41,069.01$ & 0.000112 & 0.02362 & 0.868156 & 1.001582 \\
\hline 0.006745 & 41,167 & 0.28994 & 0.28924 & $41,446.56$ & 0.000114 & 0.02384 & 0.876166 & 1.010822 \\
\hline 0.006745 & 41,305 & 0.29092 & 0.29022 & $41,585.49$ & 0.000115 & 0.02392 & 0.879139 & 1.014253 \\
\hline 0.006742 & 40,791 & 0.2873 & 0.2866 & $41,067.88$ & 0.000112 & 0.02361 & 0.868156 & 1.001358 \\
\hline 0.006743 & 40,883 & 0.28794 & 0.28725 & $41,160.55$ & 0.000113 & 0.02367 & 0.870098 & 1.003672 \\
\hline 0.006742 & 41,312 & 0.29096 & 0.29027 & $41,592.42$ & 0.000115 & 0.02392 & 0.87926 & 1.014166 \\
\hline 0.006743 & 41,445 & 0.2919 & 0.2912 & $41,726.36$ & 0.000116 & 0.02399 & 0.882113 & 1.017531 \\
\hline 0.006742 & 40,801 & 0.28737 & 0.28667 & $41,077.95$ & 0.000112 & 0.02362 & 0.868369 & 1.001603 \\
\hline 0.006742 & 40,797 & 0.28734 & 0.28664 & $41,073.92$ & 0.000112 & 0.02362 & 0.868278 & 1.001498 \\
\hline 0.006742 & 41,325 & 0.29106 & 0.29036 & $41,605.5$ & 0.000115 & 0.02392 & 0.879564 & 1.014516 \\
\hline 0.006742 & 41,464 & 0.29204 & 0.29134 & $41,745.45$ & 0.000116 & 0.024 & 0.882537 & 1.017945 \\
\hline 0.006746 & 40,290 & 0.28377 & 0.28308 & $40,563.64$ & 0.000109 & 0.02333 & 0.857448 & 0.989302 \\
\hline 0.006742 & 40,337 & 0.28409 & 0.28341 & $40,610.8$ & 0.00011 & 0.02335 & 0.858419 & 0.990126 \\
\hline 0.006746 & 40,900 & 0.28806 & 0.28737 & $41,177.79$ & 0.000113 & 0.02368 & 0.870462 & 1.004317 \\
\hline 0.006746 & 41,062 & 0.28921 & 0.28851 & $41,340.89$ & 0.000114 & 0.02378 & 0.873951 & 1.008342 \\
\hline 0.006744 & 40,368 & 0.28431 & 0.28363 & $40,642.09$ & 0.00011 & 0.02337 & 0.859086 & 0.991044 \\
\hline 0.006745 & 40,469 & 0.28503 & 0.28434 & $40,743.82$ & 0.00011 & 0.02343 & 0.86127 & 0.993637 \\
\hline 0.006744 & 40,978 & 0.28861 & 0.28792 & $41,256.23$ & 0.000113 & 0.02372 & 0.87213 & 1.006092 \\
\hline 0.006744 & 41,113 & 0.28956 & 0.28887 & $41,392.15$ & 0.000114 & 0.0238 & 0.875013 & 1.009417 \\
\hline 0.006745 & 40,131 & 0.28264 & 0.28196 & $40,403.52$ & 0.000108 & 0.02323 & 0.85402 & 0.985274 \\
\hline 0.006745 & 40,250 & 0.28348 & 0.2828 & $40,523.33$ & 0.000109 & 0.0233 & 0.856568 & 0.988213 \\
\hline 0.006744 & 40,771 & 0.28715 & 0.28646 & $41,047.83$ & 0.000112 & 0.0236 & 0.867701 & 1.000982 \\
\hline 0.006744 & 40,947 & 0.28839 & 0.2877 & $41,225.02$ & 0.000113 & 0.02371 & 0.871463 & 1.005322 \\
\hline 0.006745 & 40,289 & 0.28376 & 0.28307 & $40,562.59$ & 0.000109 & 0.02333 & 0.857418 & 0.989193 \\
\hline 0.006746 & 40,398 & 0.28453 & 0.28384 & $40,672.38$ & 0.00011 & 0.02339 & 0.859753 & 0.991962 \\
\hline 0.006745 & 40,913 & 0.28815 & 0.28746 & $41,190.83$ & 0.000113 & 0.02369 & 0.870735 & 1.004557 \\
\hline 0.006746 & 41,070 & 0.28926 & 0.28857 & $41,348.94$ & 0.000114 & 0.02378 & 0.874102 & 1.008517 \\
\hline 0.006743 & 41,763 & 0.29414 & 0.29344 & $42,046.52$ & 0.000117 & 0.02418 & 0.88891 & 1.025372 \\
\hline 0.006744 & 41,819 & 0.29454 & 0.29383 & $42,102.94$ & 0.000118 & 0.02421 & 0.890123 & 1.026849 \\
\hline 0.006744 & 42,137 & 0.29677 & 0.29606 & $42,423.1$ & 0.00012 & 0.0244 & 0.896891 & 1.034656 \\
\hline 0.006745 & 42,216 & 0.29733 & 0.29662 & $42,502.68$ & 0.00012 & 0.02444 & 0.898591 & 1.036694 \\
\hline
\end{tabular}


C. O. Arimie et al.

Continued

\begin{tabular}{|c|c|c|c|c|c|c|c|c|}
\hline 0.006744 & 41,738 & 0.29396 & 0.29326 & $42,021.39$ & 0.000117 & 0.02416 & 0.888363 & 1.024818 \\
\hline 0.006745 & 41,796 & 0.29437 & 0.29367 & $42,079.83$ & 0.000118 & 0.0242 & 0.889608 & 1.02633 \\
\hline 0.006744 & 42,122 & 0.29667 & 0.29596 & 42,408 & 0.00012 & 0.02439 & 0.896587 & 1.034306 \\
\hline 0.006745 & 42,201 & 0.29722 & 0.29651 & $42,487.58$ & 0.00012 & 0.02443 & 0.898257 & 1.036309 \\
\hline 0.006744 & 41,673 & 0.29351 & 0.2928 & $41,955.95$ & 0.000117 & 0.02413 & 0.886998 & 1.023243 \\
\hline 0.006745 & 41,737 & 0.29395 & 0.29325 & $42,020.43$ & 0.000117 & 0.02417 & 0.888333 & 1.02486 \\
\hline 0.006745 & 42,080 & 0.29638 & 0.29567 & $42,365.76$ & 0.000119 & 0.02436 & 0.895707 & 1.033367 \\
\hline 0.006745 & 42,187 & 0.29713 & 0.29642 & $42,473.48$ & 0.00012 & 0.02443 & 0.897984 & 1.035993 \\
\hline 0.006755 & 39,592 & 0.27885 & 0.27817 & $39,861.26$ & 0.000106 & 0.02294 & 0.842526 & 0.972738 \\
\hline 0.006756 & 39,684 & 0.2795 & 0.27882 & $39,953.93$ & 0.000106 & 0.023 & 0.844497 & 0.975086 \\
\hline 0.006753 & 40,354 & 0.28422 & 0.28353 & $40,628.36$ & 0.00011 & 0.02338 & 0.858813 & 0.991394 \\
\hline 0.006755 & 40,433 & 0.28477 & 0.28409 & $40,707.98$ & 0.00011 & 0.02343 & 0.860481 & 0.993468 \\
\hline 0.006759 & 39,000 & 0.27468 & 0.27402 & $39,265.39$ & 0.000103 & 0.0226 & 0.829881 & 0.958424 \\
\hline 0.00676 & 39,122 & 0.27554 & 0.27487 & $39,388.26$ & 0.000103 & 0.02268 & 0.832488 & 0.961507 \\
\hline 0.006757 & 40,008 & 0.28178 & 0.2811 & $40,280.17$ & 0.000108 & 0.02318 & 0.851412 & 0.983144 \\
\hline 0.006759 & 40,081 & 0.2823 & 0.28161 & $40,353.75$ & 0.000108 & 0.02323 & 0.852989 & 0.985112 \\
\hline 0.006762 & 38,009 & 0.2677 & 0.26705 & $38,267.77$ & 0.000098 & 0.02203 & 0.808719 & 0.934193 \\
\hline 0.006763 & 37,945 & 0.26725 & 0.2666 & $38,203.37$ & 0.000097 & 0.022 & 0.807355 & 0.932687 \\
\hline 0.006757 & 39,036 & 0.27494 & 0.27427 & $39,301.56$ & 0.000103 & 0.02262 & 0.830669 & 0.959191 \\
\hline 0.00676 & 39,092 & 0.27533 & 0.27466 & $39,358.06$ & 0.000103 & 0.02266 & 0.831852 & 0.960772 \\
\hline 0.006754 & 37,078 & 0.26114 & 0.2605 & $37,330.13$ & 0.000093 & 0.02148 & 0.788836 & 0.910682 \\
\hline 0.006755 & 37,257 & 0.26241 & 0.26177 & $37,510.38$ & 0.000094 & 0.02159 & 0.792685 & 0.915194 \\
\hline 0.006748 & 38,477 & 0.271 & 0.27034 & $38,738.41$ & 0.0001 & 0.02228 & 0.818723 & 0.944763 \\
\hline 0.006752 & 38,514 & 0.27126 & 0.2706 & $38,775.81$ & 0.0001 & 0.02231 & 0.819511 & 0.945955 \\
\hline 0.006725 & 36,796 & 0.25915 & 0.25852 & $37,045.13$ & 0.000091 & 0.02127 & 0.782805 & 0.901765 \\
\hline 0.006729 & 39,052 & 0.27504 & 0.27438 & $39,316.56$ & 0.000102 & 0.02258 & 0.830972 & 0.957538 \\
\hline 0.006718 & 40,338 & 0.2841 & 0.28341 & $40,610.82$ & 0.000109 & 0.02331 & 0.858449 & 0.988385 \\
\hline 0.006722 & 40,434 & 0.28478 & 0.28409 & $40,707.64$ & 0.00011 & 0.02337 & 0.860512 & 0.991057 \\
\hline 0.006756 & 33,988 & 0.23938 & 0.23879 & $34,219.18$ & 0.000078 & 0.01969 & 0.722917 & 0.834705 \\
\hline 0.00676 & 34,268 & 0.24135 & 0.24076 & $34,501.23$ & 0.000079 & 0.01986 & 0.728882 & 0.841844 \\
\hline 0.006739 & 36,267 & 0.25543 & 0.2548 & $36,513.06$ & 0.000089 & 0.02099 & 0.771533 & 0.88971 \\
\hline 0.006755 & 36,047 & 0.25389 & 0.25326 & $36,292.15$ & 0.000088 & 0.02089 & 0.766867 & 0.885386 \\
\hline 0.006687 & 36,451 & 0.25672 & 0.25609 & $36,696.39$ & 0.000089 & 0.02101 & 0.775442 & 0.890738 \\
\hline 0.006688 & 36,579 & 0.25762 & 0.25699 & $36,825.29$ & 0.000089 & 0.02109 & 0.778169 & 0.893938 \\
\hline
\end{tabular}


C. O. Arimie et al.

Continued

\begin{tabular}{|c|c|c|c|c|c|c|c|c|}
\hline 0.006682 & 38,170 & 0.26883 & 0.26818 & $38,426.77$ & 0.000097 & 0.022 & 0.812145 & 0.932546 \\
\hline 0.00669 & 38,131 & 0.26855 & 0.2679 & $38,387.81$ & 0.000097 & 0.02199 & 0.811296 & 0.932133 \\
\hline 0.006661 & 36,637 & 0.25803 & 0.2574 & $36,882.68$ & 0.000089 & 0.02108 & 0.779411 & 0.893544 \\
\hline 0.006663 & 37,559 & 0.26452 & 0.26387 & $37,810.93$ & 0.000094 & 0.02161 & 0.79908 & 0.916231 \\
\hline 0.00666 & 39,104 & 0.2754 & 0.27473 & $39,366.18$ & 0.000102 & 0.0225 & 0.832064 & 0.953834 \\
\hline 0.006671 & 38,900 & 0.27397 & 0.2733 & $39,161.24$ & 0.000101 & 0.0224 & 0.827728 & 0.949652 \\
\hline 0.006726 & 34,804 & 0.24512 & 0.24452 & $35,039.68$ & 0.000081 & 0.02012 & 0.7403 & 0.852864 \\
\hline 0.006733 & 34,986 & 0.24641 & 0.2458 & $35,223.16$ & 0.000082 & 0.02024 & 0.744208 & 0.857815 \\
\hline 0.006704 & 37,329 & 0.26291 & 0.26227 & $37,580.94$ & 0.000093 & 0.02155 & 0.7942 & 0.913452 \\
\hline 0.006738 & 36,706 & 0.25852 & 0.25789 & 36,955 & 0.000091 & 0.02124 & 0.780896 & 0.90044 \\
\hline 0.006792 & 32,899 & 0.23171 & 0.23114 & $33,123.98$ & 0.000073 & 0.01911 & 0.699693 & 0.810055 \\
\hline 0.006802 & 33,840 & 0.23835 & 0.23776 & $34,071.76$ & 0.000078 & 0.01968 & 0.719798 & 0.833948 \\
\hline 0.006744 & 27,684 & 0.19498 & 0.19449 & $27,871.97$ & 0.000052 & 0.01603 & 0.58856 & 0.678964 \\
\hline 0.006793 & 36,093 & 0.25421 & 0.25359 & $36,339.86$ & 0.000088 & 0.02097 & 0.767837 & 0.889013 \\
\hline 0.006629 & 36,810 & 0.25924 & 0.2586 & $37,055.64$ & 0.00009 & 0.02112 & 0.783078 & 0.895574 \\
\hline 0.006634 & 37,542 & 0.2644 & 0.26376 & $37,792.72$ & 0.000093 & 0.02155 & 0.798716 & 0.913805 \\
\hline 0.006622 & 39,906 & 0.28105 & 0.28037 & $40,172.02$ & 0.000105 & 0.02289 & 0.849198 & 0.970676 \\
\hline 0.006645 & 39,487 & 0.2781 & 0.27742 & $39,751.15$ & 0.000103 & 0.02269 & 0.840251 & 0.962127 \\
\hline 0.006953 & 23,171 & 0.16321 & 0.16279 & $23,333.24$ & 0.000037 & 0.01362 & 0.492526 & 0.576977 \\
\hline 0.006927 & 21,289 & 0.14995 & 0.14957 & $21,437.5$ & 0.000031 & 0.01249 & 0.452466 & 0.529049 \\
\hline 0.006845 & 25,843 & 0.18203 & 0.18156 & $26,021.11$ & 0.000046 & 0.01507 & 0.549405 & 0.638556 \\
\hline 0.006827 & 26,589 & 0.18728 & 0.1868 & $26,771.77$ & 0.000048 & 0.01549 & 0.565277 & 0.656133 \\
\hline 0.006765 & 19,580 & 0.13791 & 0.13755 & $19,713.36$ & 0.000026 & 0.01135 & 0.416102 & 0.480768 \\
\hline 0.006743 & 18,337 & 0.12915 & 0.12882 & $18,461.49$ & 0.000023 & 0.01061 & 0.389649 & 0.449467 \\
\hline 0.006684 & 23,331 & 0.16431 & 0.16389 & $23,487.99$ & 0.000036 & 0.01344 & 0.49585 & 0.569446 \\
\hline 0.006671 & 24,251 & 0.17079 & 0.17036 & $24,413.86$ & 0.000039 & 0.01396 & 0.515432 & 0.591355 \\
\hline 0.00667 & 13,968 & 0.09838 & 0.09812 & $14,061.79$ & 0.000013 & 0.00804 & 0.296766 & 0.340454 \\
\hline 0.006646 & 12,997 & 0.09153 & 0.0913 & $13,083.96$ & 0.000011 & 0.00747 & 0.276094 & 0.316165 \\
\hline 0.006593 & 18,912 & 0.13319 & 0.13285 & $19,037.51$ & 0.000024 & 0.01082 & 0.401848 & 0.458319 \\
\hline 0.006586 & 19,856 & 0.13983 & 0.13947 & $19,987.64$ & 0.000026 & 0.01136 & 0.4219 & 0.480932 \\
\hline 0.006619 & 10,696 & 0.07533 & 0.07513 & $10,767.27$ & 0.000008 & 0.00613 & 0.227213 & 0.259657 \\
\hline 0.00659 & 10,074 & 0.07094 & 0.07076 & $10,140.83$ & 0.000007 & 0.00576 & 0.213969 & 0.243981 \\
\hline 0.006538 & 16,118 & 0.11351 & 0.11321 & $16,224.07$ & 0.000017 & 0.00918 & 0.342432 & 0.38891 \\
\hline 0.006522 & 17,562 & 0.12367 & 0.12335 & $17,677.29$ & 0.00002 & 0.00999 & 0.373104 & 0.423223 \\
\hline
\end{tabular}


C. O. Arimie et al.

Continued

\begin{tabular}{|c|c|c|c|c|c|c|c|c|}
\hline 0.006703 & 11,014 & 0.07757 & 0.07737 & $11,088.33$ & 0.000008 & 0.00636 & 0.233972 & 0.269083 \\
\hline 0.006669 & 10,657 & 0.07506 & 0.07486 & $10,728.55$ & 0.000008 & 0.00613 & 0.226399 & 0.259708 \\
\hline 0.006593 & 16,785 & 0.11821 & 0.1179 & $16,896.4$ & 0.000019 & 0.00961 & 0.35662 & 0.406735 \\
\hline 0.006567 & 19,595 & 0.13799 & 0.13764 & $19,724.53$ & 0.000025 & 0.01119 & 0.416343 & 0.473908 \\
\hline 0.006717 & 7039 & 0.04958 & 0.04945 & 7086.601 & 0.000003 & 0.00407 & 0.149534 & 0.172154 \\
\hline 0.006675 & 8335 & 0.0587 & 0.05855 & 8391.01 & 0.000005 & 0.0048 & 0.177044 & 0.203184 \\
\hline 0.006593 & 13,533 & 0.09531 & 0.09506 & $13,622.82$ & 0.000012 & 0.00774 & 0.287501 & 0.327903 \\
\hline 0.006564 & 16,353 & 0.11517 & 0.11487 & $16,461.05$ & 0.000018 & 0.00934 & 0.347443 & 0.39539 \\
\hline 0.006679 & 13,040 & 0.09184 & 0.0916 & $13,127.68$ & 0.000011 & 0.00751 & 0.27703 & 0.318028 \\
\hline 0.00665 & 12,586 & 0.08864 & 0.08841 & $12,670.26$ & 0.000011 & 0.00723 & 0.267373 & 0.306271 \\
\hline 0.006719 & 13,070 & 0.09205 & 0.09181 & $13,158.41$ & 0.000011 & 0.00755 & 0.277663 & 0.319715 \\
\hline 0.006545 & 21,679 & 0.15267 & 0.15228 & $21,821.82$ & 0.000031 & 0.01236 & 0.460683 & 0.523493 \\
\hline 0.006759 & 7056 & 0.0497 & 0.04957 & 7104.016 & 0.000003 & 0.00409 & 0.149896 & 0.173113 \\
\hline 0.006719 & 8117 & 0.05717 & 0.05702 & 8171.907 & 0.000004 & 0.00469 & 0.172428 & 0.198542 \\
\hline 0.006647 & 14,072 & 0.09911 & 0.09885 & $14,166.16$ & 0.000013 & 0.00809 & 0.298969 & 0.342385 \\
\hline 0.006587 & 18,224 & 0.12835 & 0.12801 & $18,344.84$ & 0.000022 & 0.01042 & 0.387234 & 0.441449 \\
\hline 0.006523 & 8069 & 0.05682 & 0.05668 & 8121.98 & 0.000004 & 0.00459 & 0.171373 & 0.194408 \\
\hline 0.006631 & 12,589 & 0.08866 & 0.08843 & $12,673.03$ & 0.00001 & 0.00723 & 0.267434 & 0.305899 \\
\hline 0.006401 & 14,479 & 0.10196 & 0.10169 & $14,572.28$ & 0.000013 & 0.00816 & 0.30757 & 0.345614 \\
\hline 0.016748 & $-110,431$ & -0.78172 & -0.78092 & $-112,312$ & 0.002082 & -0.10192 & -2.3926 & 4.371676 \\
\hline 0.006547 & 18,806 & 0.13244 & 0.1321 & $18,929.93$ & 0.000023 & 0.01072 & 0.399584 & 0.454133 \\
\hline 0.006306 & 5064 & 0.03566 & 0.03556 & 5096.136 & 0.000002 & 0.00283 & 0.107548 & 0.119944 \\
\hline 0.00625 & 13,374 & 0.09417 & 0.09393 & $13,458.11$ & 0.000011 & 0.00745 & 0.284061 & 0.315385 \\
\hline 0.00625 & 19,343 & 0.1362 & 0.13585 & $19,464.65$ & 0.000023 & 0.01077 & 0.410938 & 0.456253 \\
\hline 0.006993 & 15,273 & 0.10758 & 0.10731 & $15,380.56$ & 0.000016 & 0.00901 & 0.324533 & 0.381278 \\
\hline 0.006223 & 4129 & 0.02907 & 0.029 & 4154.856 & 0.000001 & 0.00229 & 0.087672 & 0.097128 \\
\hline 0.006183 & 15,727 & 0.11074 & 0.11045 & $15,824.85$ & 0.000015 & 0.00871 & 0.334071 & 0.368904 \\
\hline 0.006192 & 21,843 & 0.1538 & 0.15341 & $21,979.09$ & 0.000029 & 0.01211 & 0.464096 & 0.512862 \\
\hline 0.006049 & 29,116 & 0.205 & 0.20448 & $29,293.19$ & 0.000051 & 0.01595 & 0.618865 & 0.675901 \\
\hline 0.005951 & 23,060 & 0.16235 & 0.16194 & $23,198.05$ & 0.000032 & 0.01253 & 0.489928 & 0.530703 \\
\hline 0.005997 & 31,983 & 0.22518 & 0.22462 & $32,175.96$ & 0.000061 & 0.01745 & 0.679926 & 0.739372 \\
\hline 0.005974 & 40,856 & 0.28764 & 0.28695 & $41,101.54$ & 0.000099 & 0.02225 & 0.869188 & 0.943355 \\
\hline 0.006387 & -5515 & -0.03884 & -0.03873 & -5550.45 & 0.000002 & -0.00311 & -0.11714 & 0.131483 \\
\hline 0.006213 & -2138 & -0.01505 & -0.01502 & -2151.37 & 0 & -0.00119 & -0.04539 & 0.050243 \\
\hline
\end{tabular}


C. O. Arimie et al.

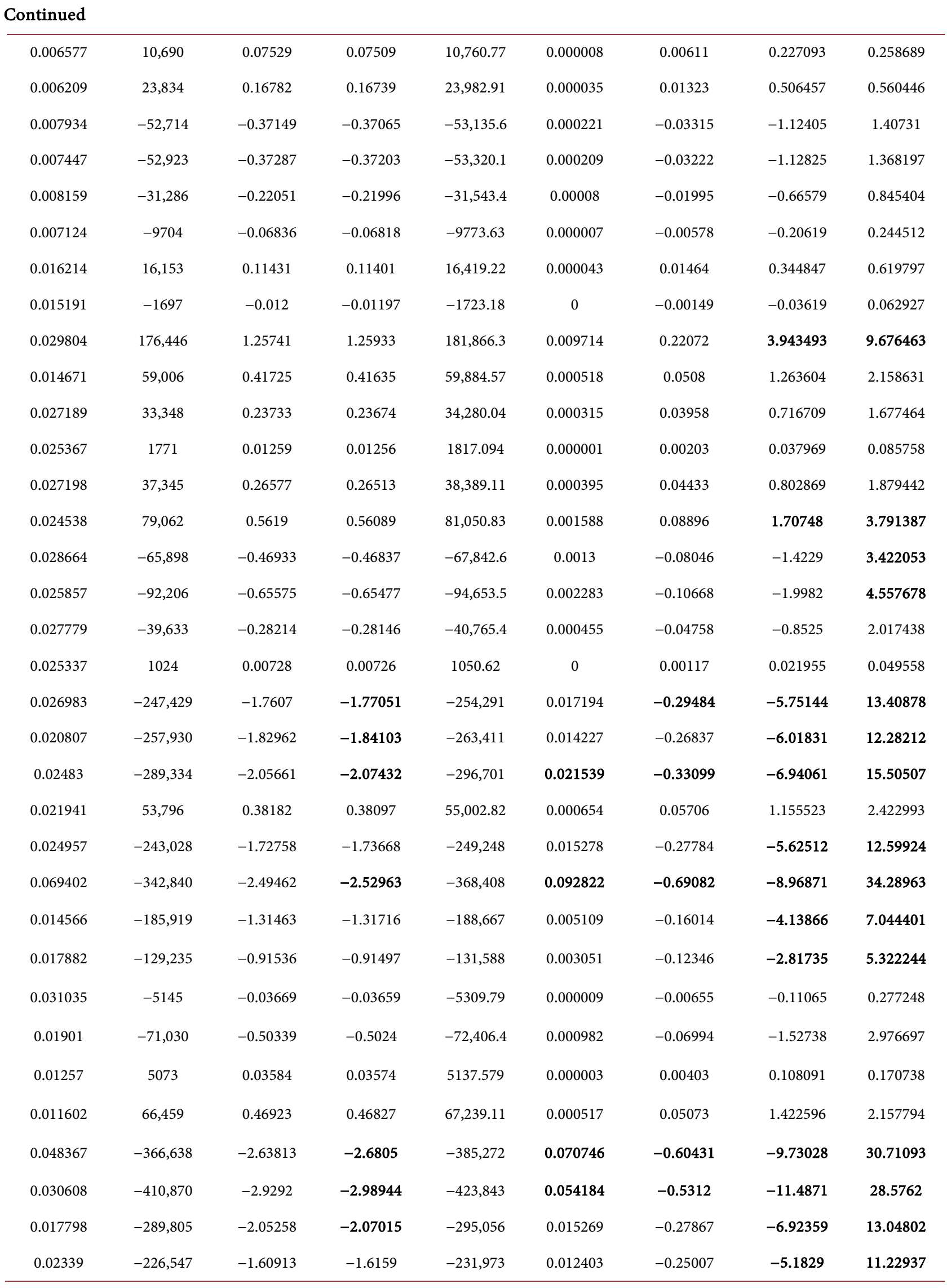


C. O. Arimie et al.

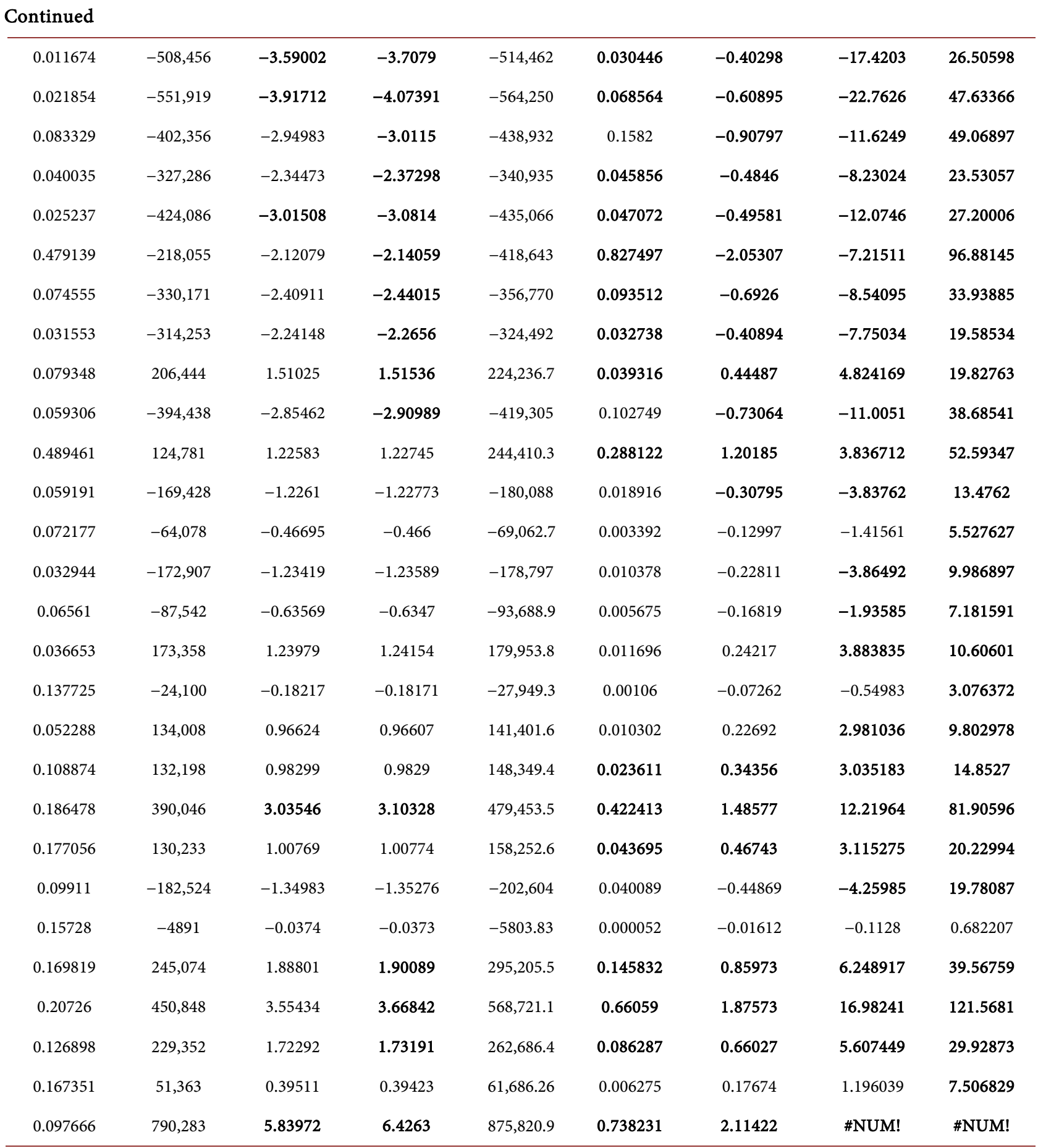

Footnote: $d_{i}>3 ; t_{0.05}(193)=1.646 ; 4 / n=4 / 193=0.0204 ; 2 \sqrt{\frac{k}{n}}=0.286 ; t_{0.05}(193)=1.646 ; A_{i}>3$.

Table 8. Summary of results in Table 7.

\begin{tabular}{ccccccc}
\hline $\begin{array}{c}\text { Outlier detection } \\
\text { methods }\end{array}$ & $\begin{array}{c}\text { Standardized } \\
\text { residuals } \\
\varepsilon_{S T . i}\end{array}$ & $\begin{array}{c}\text { Studentised } \\
\text { residuals } \\
\varepsilon_{S, i}\end{array}$ & $\begin{array}{c}\text { Cook's } \\
\text { distance } \\
D_{i}\end{array}$ & $\begin{array}{c}\text { Different-in-fits } \\
\text { (DFFITS) } \\
\text { DFFit }\end{array}$ & $\begin{array}{c}\text { Jack-knife } \\
\text { residuals }\end{array}$ & $\begin{array}{c}\text { Atkinson's } \\
\text { measure } \\
\varepsilon_{J . i}\end{array}$ \\
\hline Numbers of outliers & $\mathbf{5}$ & $\mathbf{2 0}$ & $\mathbf{2 1}$ & $\mathbf{2 5}$ & $\mathbf{3 9}$ & $\mathbf{3 2}$ \\
\hline
\end{tabular}




\section{Graphical Methods}

Figures 4-10 show the results of graphical methods.

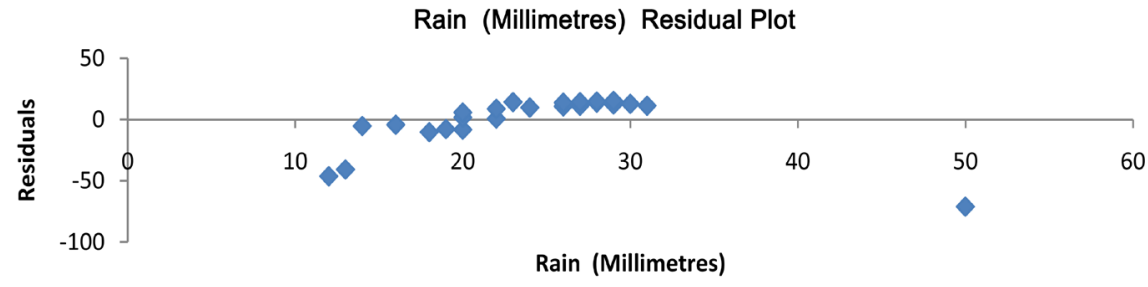

Figure 4. Graph of the predicted residuals.

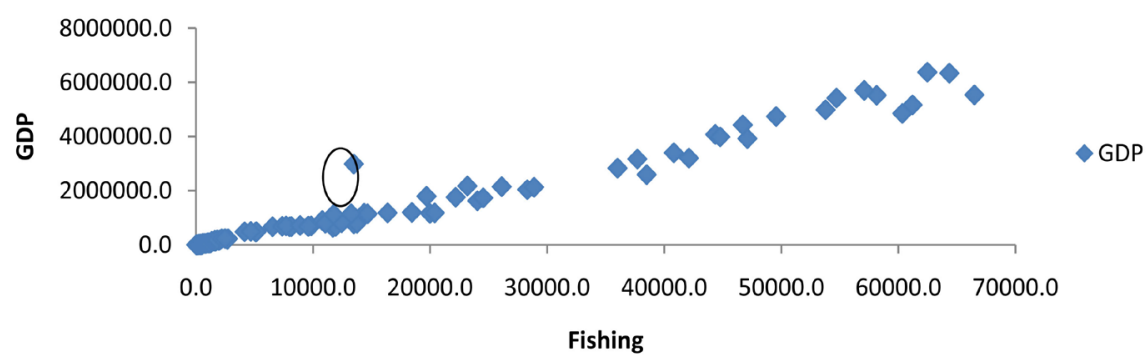

Figure 5. Scatter plot of Fishing against GDP.

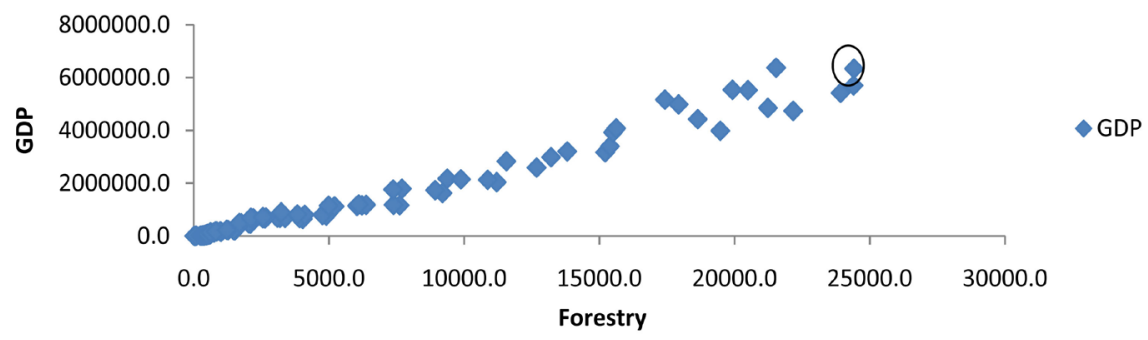

Figure 6. Forestry against GDP.

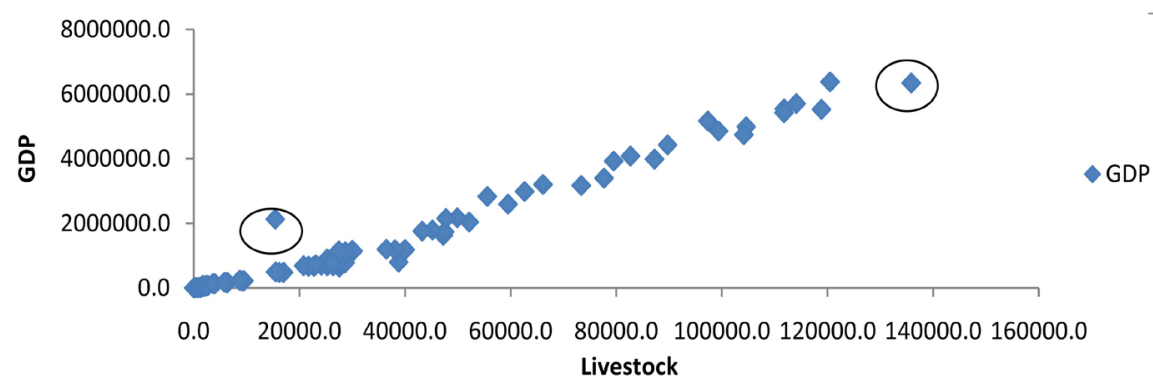

Figure 7. Livestock against GDP.

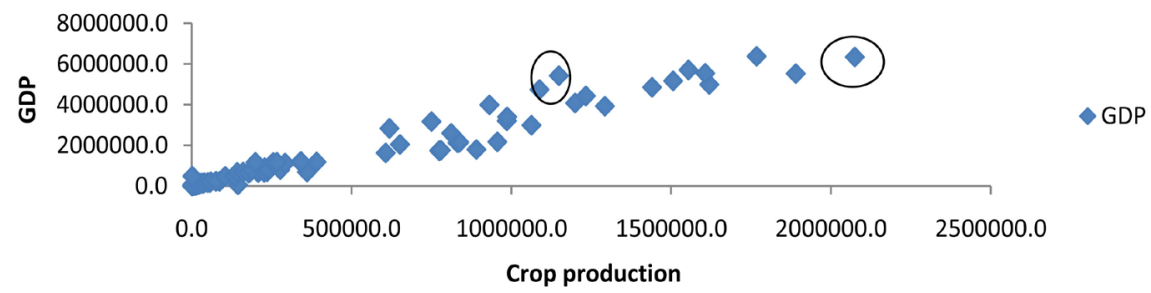

Figure 8. Crop production against GDP. 
Fishing Residual Plot
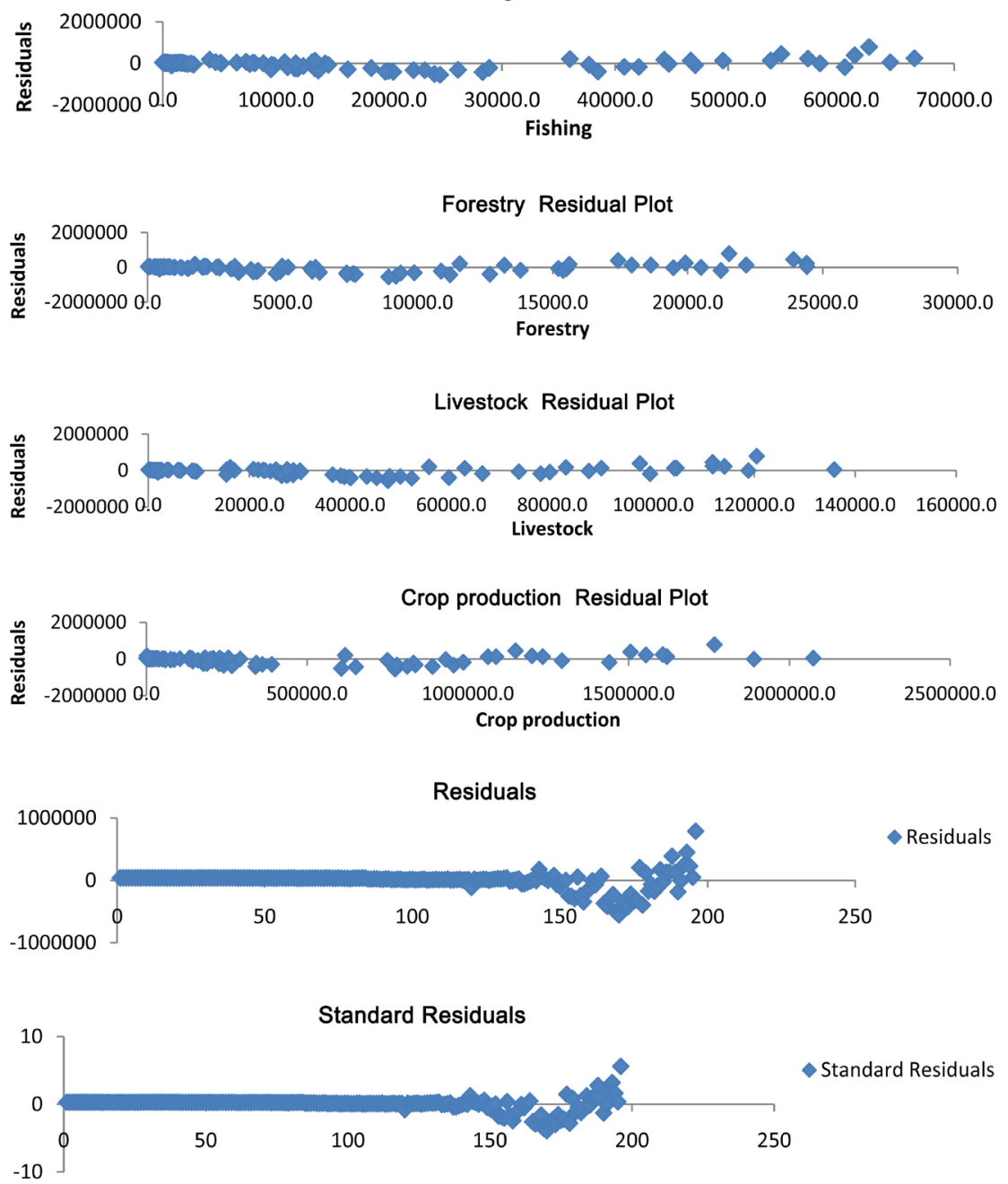

Figure 9. Graphs of predicted residuals.
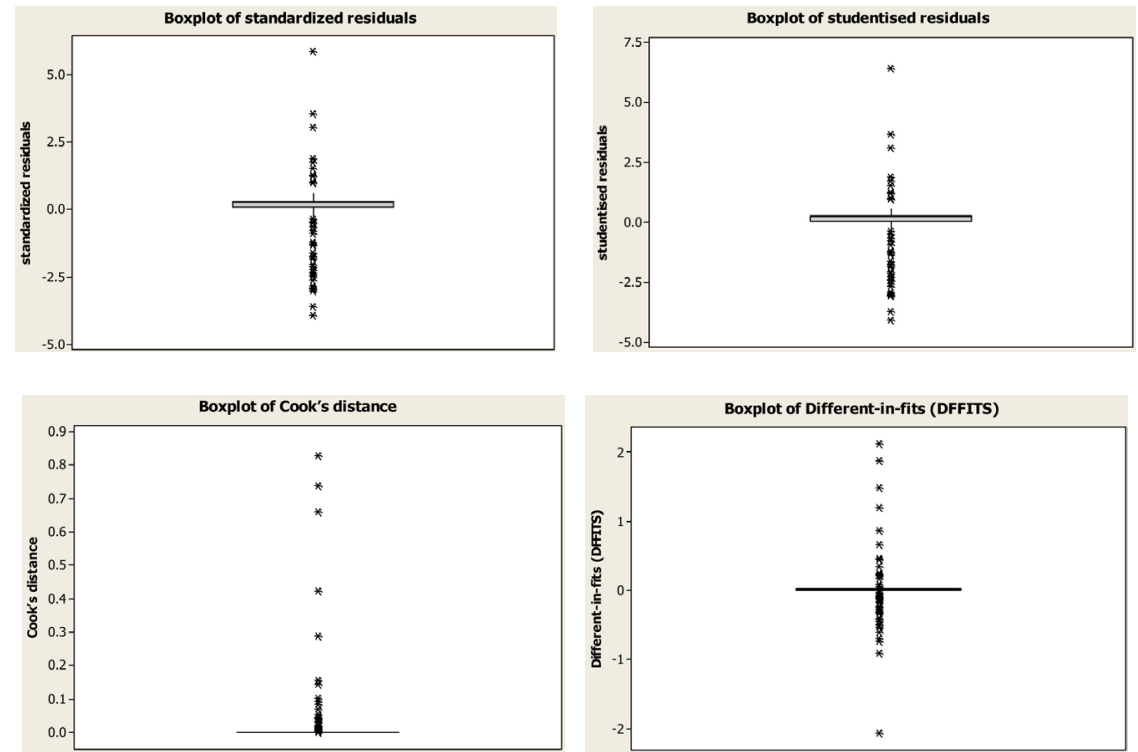

Figure 10. Boxplots of outliers detected by the analytical methods indicated. 


\section{Conclusion}

Outliers detection and effects on simple and multiple linear regression modeling were studied using the above listed analytical and graphical methods. Two data sets were used for the illustration. From the results obtained, we concluded that by removing the influential point (or Outliers), the model adequacy increased (from $\mathrm{R}^{2}=0.72$ to $\mathrm{R}^{2}=0.97$ ). Also, Jack-knife residuals and Atkinson's measure methods are more useful for detecting outliers.

\section{Acknowledgements}

We thank the National Bureau of Statistics and the Central Bank of Nigeria (CBN) for granting us access to their statistical bulletins from which the data used were extracted.

\section{Conflicts of Interest}

The authors declare no conflicts of interest regarding the publication of this paper.

\section{References}

[1] Bollen, K.A. and Jackman, R.W. (1990) Regression Diagnostics: An Expository Treatment of Outliers and Influential Cases. In: Fox, J. and Scott, L.J., Eds., Modern Methods of Data Analysis, Sage, Newbury Park, 257-291.

[2] Hawkins, D.M. (1983) Discussion of Paper by Beckman and Cook. Technometrics, 25, 155-156. https://doi.org/10.1080/00401706.1983.10487843

[3] Dixon, W.J. (1950) Analysis of Extreme Values. The Annals of Mathematical Statistics, 21, 488-506. https://doi.org/10.1214/aoms/1177729747

[4] Barnett, V. and Lewis, T. (1994) Outliers in Statistical Data. John Wiley, New York.

[5] Montgomery, D.C., Peck, E.A. and Vining, G.G. (2013) Introduction to Linear Regression Analysis. John Wiley and Sons, Hoboken.

[6] Abuzaid, A.H., Hussin, A.G. and Mohamed, I.B. (2008) Identifying Single Outlier in Linear Circular Regression Model Based on Circular Distance. Journal of Applied Probability and Statistics, 3, 107-117.

[7] Zhang, Y., Meratnia, N. and Havinga, P.J.M. (2010) Outlier Detection Techniques for Wireless Sensor Networks Survey. IEEE Communication Survey and Tutorial, 12, 159-170. https://doi.org/10.1109/SURV.2010.021510.00088

[8] Rousseeuw, P.J. (1984) Least Median of Squares Regression. Journal of the American Statistical Association, 79, 871-880. https://doi.org/10.1080/01621459.1984.10477105

[9] Aggarwal, C.C. and Yu, P.S. (2013) Outlier Detection for High Dimensional Data. https://www.researchgate.net/publication/2401320_Outlier_Detection_for_High_Di mensional_Data

[10] Arning, A., Agrawal, R. and Raghavan, P. (1996) A Linear Method for Deviation Detection in Large Databases. KDD-1996, Portland, 2-4 August 1996, 164-169.

[11] Hodge, V.J. and Austin, J. (2018) An Evaluation of Classification and Outlier Detection Algorithms. https://arxiv.org/pdf/1805.00811.pdf

[12] Sebert, D.M., Montgomery, D.C. and Rollier, D.A. (1998) Clustering Algorithm for 
Identifying Multiple Outliers in Linear Regression. Computational Statistics and Data Analysis, 27, 461-484. https://doi.org/10.1016/S0167-9473(98)00021-8

[13] Worden, K., Manson, G. and Fieller, N.R.J. (2000) Damage Detection Using Outlier Analysis. Journal of Sound and Vibration, 229, 647-667. https://doi.org/10.1006/jsvi.1999.2514

[14] Kitagawa, G. (1984) Bayesian Analysis of Outliers via Akaike's Predictive Likelihood of a Model. Communication Statistics-Simulation Computation, 13, 107-126. https://doi.org/10.1080/03610918408812361

[15] Fung, W.-K. and Bacon-Shone, J. (1993) Quasi-Bayesian Modelling of Multivariate Outliers. Computational Statistics and Data Analysis, 16, 271-278. https://doi.org/10.1016/0167-9473(93)90129-H

[16] Belsey, D.A., Kuh, E. and Welsch, R.E. (1980) Regression Diagnostics: Identifying Influential Data and Sources of Collinearity. John Wiley, Hoboken. https://doi.org/10.1002/0471725153

[17] Cook, R.D. (1977) Detection of Influential Observation in Linear Regression. Technometrics, 19, 15-18. https://doi.org/10.1080/00401706.1977.10489493

[18] Williams, D.X. (1973) Letter to the Editor. Applied Statistics, 22, 407-408. https://doi.org/10.1111/j.1467-9876.1973.tb00281.x

[19] Meloun, M. and Militky, J. (2001) Detection of Single Influential Points in OLS Regression Model Building. Analytica Chimica Acta, 439, 169-191.

https://doi.org/10.1016/S0003-2670(01)01040-6 


\section{Appendix}

Data 1.

\begin{tabular}{cccccccccccc}
\hline $\begin{array}{c}\text { Rain } \\
(\mathrm{mm})\end{array}$ & $\begin{array}{c}\text { Wheat } \\
(\mathrm{kg})\end{array}$ & $\begin{array}{c}\text { Rain } \\
(\mathrm{mm})\end{array}$ & $\begin{array}{c}\text { Wheat } \\
(\mathrm{kg})\end{array}$ & $\begin{array}{c}\text { Rain } \\
(\mathrm{mm})\end{array}$ & $\begin{array}{c}\text { Wheat } \\
(\mathrm{kg})\end{array}$ & $\begin{array}{c}\text { Rain } \\
(\mathrm{mm})\end{array}$ & $\begin{array}{c}\text { Wheat } \\
(\mathrm{kg})\end{array}$ & $\begin{array}{c}\text { Rain } \\
(\mathrm{mm})\end{array}$ & $\begin{array}{c}\text { Wheat } \\
(\mathrm{kg})\end{array}$ & $\begin{array}{c}\text { Rain } \\
(\mathrm{mm})\end{array}$ & $\begin{array}{c}\text { Wheat } \\
(\mathrm{kg})\end{array}$ \\
\hline 12 & 260 & 19 & 330 & 22 & 360 & 27 & 385 & 29 & 398 & 50 & 406 \\
13 & 270 & 20 & 334 & 23 & 370 & 27 & 388 & 30 & 400 & \\
14 & 310 & 20 & 344 & 24 & 370 & 28 & 392 & 30 & 400 & \\
16 & 320 & 20 & 348 & 26 & 380 & 28 & 393 & 31 & 403 & \\
18 & 323 & 22 & 352 & 26 & 383 & 29 & 395 & 31 & 403 & \\
\hline
\end{tabular}

Data 2.

\begin{tabular}{|c|c|c|c|c|c|c|}
\hline S/no. & Year & Crop production & Livestock & Forestry & Fishing & GDP \\
\hline 1 & \multirow{4}{*}{1961} & 224.4 & 31.0 & 37.0 & 9.4 & 497.7 \\
\hline 2 & & 288.2 & 31.2 & 35.0 & 9.6 & 558.7 \\
\hline 3 & & 329.3 & 29.7 & 31.0 & 7.7 & 586.0 \\
\hline 4 & & 288.3 & 28.4 & 29.7 & 8.7 & 580.0 \\
\hline 5 & \multirow{4}{*}{1962} & 229.2 & 35.2 & 34.6 & 11.4 & 527.1 \\
\hline 6 & & 292.4 & 35.4 & 33.6 & 11.6 & 601.5 \\
\hline 7 & & 336.4 & 33.7 & 28.9 & 9.3 & 617.2 \\
\hline 8 & & 294.5 & 32.2 & 27.7 & 10.5 & 615.5 \\
\hline 9 & \multirow{4}{*}{1963} & 257.3 & 37.0 & 34.5 & 11.7 & 575.5 \\
\hline 10 & & 328.2 & 37.2 & 33.5 & 11.9 & 561.0 \\
\hline 11 & & 377.6 & 35.4 & 28.8 & 9.6 & 680.7 \\
\hline 12 & & 330.6 & 33.9 & 27.6 & 10.8 & 680.3 \\
\hline 13 & \multirow{4}{*}{1964} & 268.6 & 33.8 & 41.0 & 11.7 & 608.7 \\
\hline 14 & & 342.6 & 43.0 & 39.8 & 12.0 & 700.9 \\
\hline 15 & & 394.2 & 32.4 & 34.3 & 9.6 & 720.9 \\
\hline 16 & & 345.1 & 31.0 & 32.9 & 10.8 & 725.4 \\
\hline 17 & \multirow{4}{*}{1965} & 262.4 & 38.1 & 38.9 & 18.2 & 648.6 \\
\hline 18 & & 334.8 & 38.3 & 37.8 & 18.6 & 736.2 \\
\hline 19 & & 385.2 & 36.5 & 32.5 & 14.9 & 760.9 \\
\hline 20 & & 337.2 & 34.9 & 31.2 & 16.8 & 758.7 \\
\hline 21 & \multirow{4}{*}{1966} & 260.1 & 40.1 & 41.6 & 20.7 & 700.4 \\
\hline 22 & & 331.8 & 40.3 & 40.3 & 21.2 & 789.9 \\
\hline 23 & & 381.8 & 38.4 & 34.8 & 17.0 & 799.8 \\
\hline 24 & & 334.3 & 36.1 & 33.3 & 19.1 & 820.0 \\
\hline 25 & \multirow{2}{*}{1967} & 288.9 & 40.1 & 38.4 & 28.9 & 758.4 \\
\hline 26 & & 368.5 & 40.3 & 37.2 & 29.5 & 856.4 \\
\hline
\end{tabular}




\section{Continued}

\begin{tabular}{|c|c|c|c|c|c|c|}
\hline 27 & & 423.9 & 38.4 & 32.1 & 23.6 & 869.4 \\
\hline 28 & & 371.2 & 36.1 & 30.7 & 26.6 & 890.5 \\
\hline 29 & \multirow{4}{*}{1968} & 239.8 & 34.8 & 20.3 & 30.2 & 614.9 \\
\hline 30 & & 305.9 & 34.9 & 19.7 & 30.9 & 698.8 \\
\hline 31 & & 351.9 & 33.3 & 17.0 & 24.8 & 710.0 \\
\hline 32 & & 308.1 & 31.8 & 16.3 & 27.9 & 728.9 \\
\hline 33 & \multirow{4}{*}{1969} & 217.6 & 33.8 & 20.6 & 30.7 & 594.5 \\
\hline 34 & & 277.6 & 34.0 & 20.0 & 31.4 & 675.2 \\
\hline 35 & & 319.4 & 32.4 & 17.2 & 25.2 & 680.3 \\
\hline 36 & & 279.6 & 31.0 & 16.5 & 28.4 & 705.6 \\
\hline 37 & \multirow{4}{*}{1970} & 269.8 & 37.3 & 22.0 & 34.9 & 803.2 \\
\hline 38 & & 344.2 & 37.6 & 21.3 & 35.8 & 899.1 \\
\hline 39 & & 396.0 & 35.7 & 18.4 & 28.6 & 903.1 \\
\hline 40 & & 346.7 & 34.1 & 17.6 & 32.2 & 944.0 \\
\hline 41 & \multirow{4}{*}{1971} & 392.4 & 45.6 & 40.7 & 74.3 & 1211.6 \\
\hline 42 & & 500.5 & 45.9 & 39.5 & 75.8 & 1337.3 \\
\hline 43 & & 575.9 & 43.7 & 34.1 & 60.9 & 1337.3 \\
\hline 44 & & 504.2 & 41.8 & 32.6 & 68.6 & 1339.3 \\
\hline 45 & \multirow{4}{*}{1972} & 467.5 & 49.5 & 46.0 & 86.3 & 1392.7 \\
\hline 46 & & 596.4 & 49.8 & 44.6 & 88.1 & 1556.7 \\
\hline 47 & & 686.1 & 47.4 & 38.5 & 70.7 & 1681.3 \\
\hline 48 & & 600.7 & 45.4 & 36.9 & 79.7 & 1677.1 \\
\hline 49 & \multirow{4}{*}{1973} & 449.0 & 60.3 & 56.9 & 105.2 & 1735.8 \\
\hline 50 & & 572.8 & 60.7 & 55.2 & 107.4 & 1691.3 \\
\hline 51 & & 659.0 & 57.7 & 47.6 & 86.2 & 1816.9 \\
\hline 52 & & 576.9 & 55.3 & 45.6 & 97.1 & 1794.9 \\
\hline 53 & \multirow{4}{*}{1974} & 446.7 & 86.2 & 64.3 & 119.3 & 1884.3 \\
\hline 54 & & 569.9 & 86.6 & 62.4 & 121.8 & 2071.0 \\
\hline 55 & & 655.6 & 82.4 & 53.8 & 97.8 & 2179.9 \\
\hline 56 & & 574.0 & 78.5 & 51.5 & 110.1 & 2127.1 \\
\hline 57 & \multirow{4}{*}{1975} & 545.5 & 184.8 & 45.1 & 200.8 & 2252.5 \\
\hline 58 & & 695.9 & 185.8 & 43.8 & 204.9 & 4627.2 \\
\hline 59 & & 800.6 & 176.8 & 37.7 & 164.5 & 4706.5 \\
\hline 60 & & 701.0 & 169.8 & 36.1 & 165.3 & 4528.1 \\
\hline 61 & \multirow{3}{*}{1976} & 738.6 & 202.6 & 89.6 & 279.1 & 4981.2 \\
\hline 62 & & 942.1 & 203.7 & 87.0 & 284.9 & 5352.5 \\
\hline 63 & & 1083.9 & 193.9 & 75.0 & 228.6 & 5386.7 \\
\hline
\end{tabular}




\section{Continued}

\begin{tabular}{|c|c|c|c|c|c|c|}
\hline 64 & & 949.0 & 185.6 & 71.6 & 257.6 & 5195.1 \\
\hline 65 & \multirow{4}{*}{1977} & 818.0 & 260.4 & 94.6 & 174.7 & 6530.9 \\
\hline 66 & & 1043.5 & 261.3 & 91.8 & 178.3 & 6714.7 \\
\hline 67 & & 1200.6 & 249.2 & 79.2 & 143.1 & 6663.3 \\
\hline 68 & & 1051.1 & 238.5 & 75.8 & 161.2 & 6424.7 \\
\hline 69 & \multirow{4}{*}{1978} & 1013.6 & 319.1 & 82.6 & 204.2 & 6853.1 \\
\hline 70 & & 1293.0 & 321.1 & 80.1 & 208.4 & 7928.0 \\
\hline 71 & & 1487.6 & 305.8 & 69.1 & 167.3 & 7876.2 \\
\hline 72 & & 1302.0 & 292.5 & 66.2 & 188.5 & 7515.7 \\
\hline 73 & \multirow{4}{*}{1979} & 1001.0 & 339.2 & 82.8 & 368.4 & 8099.9 \\
\hline 74 & & 1276.8 & 341.0 & 80.3 & 376.0 & 8490.9 \\
\hline 75 & & 1469.1 & 324.5 & 69.3 & 301.8 & 8636.4 \\
\hline 76 & & 1286.2 & 310.6 & 66.3 & 349.0 & 8309.1 \\
\hline 77 & \multirow{4}{*}{1980} & 1103.3 & 384.9 & 85.0 & 496.0 & 9103.7 \\
\hline 78 & & 1407.4 & 387.0 & 82.5 & 506.2 & $10,330.8$ \\
\hline 79 & & 1619.2 & 368.2 & 71.1 & 406.3 & 1486.3 \\
\hline 80 & & 1417.6 & 352.4 & 66.1 & 457.7 & $10,061.7$ \\
\hline 81 & \multirow{4}{*}{1981} & 1314.1 & 482.4 & 87.3 & 323.9 & $11,095.9$ \\
\hline 82 & & 1676.2 & 485.0 & 84.7 & 330.6 & $12,105.0$ \\
\hline 83 & & 1928.5 & 461.5 & 73.0 & 265.4 & $12,377.5$ \\
\hline 84 & & 1688.4 & 441.7 & 70.0 & 298.9 & $11,908.9$ \\
\hline 85 & \multirow{4}{*}{1982} & 2006.3 & 440.5 & 294.4 & 192.2 & $13,241.6$ \\
\hline 86 & & 2559.3 & 442.5 & 285.6 & 196.2 & $11,241.9$ \\
\hline 87 & & 2944.5 & 421.1 & 246.3 & 157.5 & $11,958.3$ \\
\hline 88 & & 2577.9 & 402.1 & 236.9 & 177.4 & $11,743.6$ \\
\hline 89 & \multirow{4}{*}{1983} & 2242.3 & 690.7 & 295.9 & 235.2 & 12,675 \\
\hline & & 2860.2 & 692.5 & 287.0 & 242.1 & $11,420.9$ \\
\hline 91 & & 3290.6 & 660.9 & 247.6 & 192.7 & $12,344.8$ \\
\hline 92 & & 2881.0 & 632.5 & 237.1 & 217.1 & $12,176.5$ \\
\hline 93 & \multirow{4}{*}{1984} & 2559.6 & 905.2 & 321.2 & 344.9 & $13,127.1$ \\
\hline 94 & & 3285.1 & 910.1 & 311.7 & 352.0 & $12,226.5$ \\
\hline 95 & & 3756.5 & 866.1 & 268.8 & 282.5 & $13,361.6$ \\
\hline 96 & & 3288.8 & 828.9 & 257.5 & 318.3 & $13,226.1$ \\
\hline 97 & \multirow{4}{*}{1985} & 3365.0 & 1153.9 & 350.3 & 303.2 & $14,293.2$ \\
\hline 98 & & 4292.6 & 1160.1 & 339.9 & 309.4 & $13,859.0$ \\
\hline 99 & & 4938.6 & 1104.0 & 293.1 & 248.4 & $15,021.6$ \\
\hline 100 & & 4323.8 & 1056.7 & 280.7 & 279.7 & $14,989.6$ \\
\hline
\end{tabular}




\section{Continued}

\begin{tabular}{|c|c|c|c|c|c|c|}
\hline 101 & \multirow{4}{*}{1986} & 3923.8 & 1248.5 & 372.5 & 188.8 & $15,750.4$ \\
\hline 102 & & 5005.1 & 1255.3 & 361.5 & 192.7 & $15,647.0$ \\
\hline 103 & & 5758.6 & 1194.5 & 311.7 & 154.6 & $17,115.3$ \\
\hline 104 & & 5041.6 & 1143.3 & 298.6 & 174.2 & $18,049.3$ \\
\hline 105 & \multirow{4}{*}{1987} & 4066.6 & 1288.0 & 399.0 & 266.6 & $15,874.4$ \\
\hline 106 & & 5186.0 & 1295.0 & 387.2 & 274.2 & $17,460.1$ \\
\hline 107 & & 5966.6 & 1232.4 & 333.8 & 220.1 & $17,418.8$ \\
\hline 108 & & 5223.8 & 1179.5 & 319.8 & 247.9 & $18,393.6$ \\
\hline 109 & \multirow{4}{*}{1988} & 6208.9 & 1459.6 & 402.5 & 232.3 & $24,995.7$ \\
\hline 110 & & 7918.8 & 1467.5 & 391.6 & 237.0 & $25,452.0$ \\
\hline 111 & & 9110.7 & 1396.6 & 387.7 & 190.2 & $25,416.5$ \\
\hline 112 & & 7976.5 & 1336.6 & 323.4 & 214.3 & $27,358.3$ \\
\hline 113 & \multirow{4}{*}{1989} & 9681.4 & 1549.6 & 472.1 & 407.3 & $32,232.7$ \\
\hline 114 & & $12,349.6$ & 1558.0 & 458.1 & 415.7 & $34,961.2$ \\
\hline 115 & & $14,208.4$ & 1482.6 & 395.1 & 333.6 & $35,329.8$ \\
\hline 116 & & $12,439.5$ & 1419.0 & 378.4 & 375.9 & $36,561.7$ \\
\hline 117 & \multirow{4}{*}{1990} & $11,252.2$ & 2055.3 & 552.0 & 843.4 & $53,255.4$ \\
\hline 118 & & $14,353.4$ & 2066.4 & 535.7 & 550.8 & $54,337.8$ \\
\hline 119 & & $16,513.8$ & 1966.4 & 462.0 & 690.9 & $53,793.9$ \\
\hline 120 & & $144,459.0$ & 1882.1 & 442.4 & 778.3 & $55,410.5$ \\
\hline 121 & \multirow{4}{*}{1991} & 1360.9 & 2465.8 & 595.5 & 1120.7 & $65,929.7$ \\
\hline 122 & & $17,357.0$ & 2479.1 & 577.9 & 1143.8 & $67,103.8$ \\
\hline 123 & & $19,969.4$ & 2359.2 & 498.3 & 918.1 & $66,260.6$ \\
\hline 124 & & $17,483.4$ & 2258.0 & 477.3 & 1034.2 & $69,255.9$ \\
\hline 125 & \multirow{4}{*}{1992} & $15,911.0$ & 1715.0 & 618.5 & 1249.5 & $75,449.4$ \\
\hline 126 & & $20,296.2$ & 2729.7 & 600.2 & 1275.2 & $75,243.7$ \\
\hline 127 & & $23,350.9$ & 2597.7 & 517.6 & 1023.6 & $77,318.5$ \\
\hline 128 & & $20,444.0$ & 2486.3 & 495.7 & 1153.1 & $80,126.2$ \\
\hline 129 & \multirow{4}{*}{1993} & $24,009.1$ & 4013.9 & 759.3 & 1647.6 & $133,927.1$ \\
\hline 130 & & $30,626.1$ & 4035.8 & 736.6 & 1681.7 & $133,280.6$ \\
\hline 131 & & $35,235.7$ & 3841.4 & 635.4 & 1348.7 & $130,710.2$ \\
\hline 132 & & $30,849.2$ & 3675.7 & 608.5 & 1520.5 & $134,716.0$ \\
\hline 133 & \multirow{4}{*}{1994} & $39,007.5$ & 6375.5 & 1006.9 & 1951.2 & $158,745.6$ \\
\hline 134 & & $49,758.2$ & 6410.0 & 977.0 & 1991.5 & $171,233.1$ \\
\hline 135 & & $57,247.3$ & 6100.0 & 842.6 & 1598.4 & $170,640.0$ \\
\hline 136 & & $50,120.6$ & 5838.3 & 806.9 & 1800.7 & $175,251.1$ \\
\hline
\end{tabular}




\section{Continued}

\begin{tabular}{|c|c|c|c|c|c|c|}
\hline 137 & \multirow{4}{*}{1995} & $59,061.5$ & 9465.7 & 1518.6 & 2681.8 & $211,794.3$ \\
\hline 138 & & $75,339.1$ & 9517.0 & 1473.5 & 2737.1 & $225,286.2$ \\
\hline 139 & & $86,678.5$ & 9056.6 & 1270.8 & 2196.9 & $227,716.4$ \\
\hline 140 & & $75,887.8$ & 8668.1 & 1217.0 & 2474.9 & $235,956.4$ \\
\hline 141 & \multirow{4}{*}{1996} & $104,606.4$ & $16,963.2$ & 2096.2 & 5087.5 & $475,135.4$ \\
\hline 142 & & $133,817.8$ & $17,035.0$ & 2033.0 & 5172.0 & $482,976.8$ \\
\hline 143 & & 1539.8 & $16,210.9$ & 1753.3 & 4151.2 & $481,117.3$ \\
\hline 144 & & $134,792.4$ & $15,515.5$ & 1679.0 & 4676.6 & $493,962.0$ \\
\hline 145 & \multirow{4}{*}{1997} & $141,959.5$ & $22,731.2$ & 2632.0 & 7979.1 & $670,619.8$ \\
\hline 146 & & $181,084.2$ & $22,854.3$ & 2554.0 & 8143.7 & $675,141.6$ \\
\hline 147 & & $208,339.4$ & $21,748.8$ & 2202.5 & 6536.4 & $670,697.4$ \\
\hline 148 & & $182,403.0$ & $20,815.9$ & 2109.3 & 7363.6 & $686,280.4$ \\
\hline 149 & \multirow{4}{*}{1998} & $160,649.3$ & $25,279.9$ & 3186.9 & 9635.5 & $686,351.0$ \\
\hline 150 & & $204,924.9$ & $25,416.8$ & 3092.0 & 9834.4 & $700,532.3$ \\
\hline 151 & & $235,788.3$ & $24,187.3$ & 2666.9 & 7693.4 & $699,923.5$ \\
\hline 152 & & $206,417.3$ & $23,149.8$ & 2554.0 & 8892.3 & $715,165.8$ \\
\hline 153 & \multirow{4}{*}{1999} & $177,413.6$ & $27,595.5$ & 4031.4 & $11,685.8$ & $647,960.4$ \\
\hline 154 & & $226,309.6$ & $27,345.0$ & 3911.9 & $11,928.9$ & $678,288.7$ \\
\hline 155 & & $360,371.7$ & $26,402.9$ & 3373.6 & 9572.9 & $685,015.5$ \\
\hline 156 & & $227,957.8$ & $25,270.3$ & 3230.8 & $10,784.4$ & $897,156.2$ \\
\hline 157 & \multirow{4}{*}{2000} & $188,577.0$ & $28,651.9$ & 4900.6 & $13,478.6$ & $777,923.9$ \\
\hline 158 & & $240,549.6$ & $38,807.0$ & 4755.3 & $13,756.7$ & $799,246.2$ \\
\hline 159 & & $276,755.0$ & $27,413.5$ & 4101.0 & $11,041.0$ & $801,411.1$ \\
\hline 160 & & $242,301.5$ & $26,237.6$ & 3827.4 & $12,438.9$ & $816,338.7$ \\
\hline 161 & \multirow{4}{*}{2001} & $198,896.3$ & $30,014.3$ & 6217.6 & $14,364.2$ & $1,165,093.3$ \\
\hline 162 & & $253,714.9$ & $30,176.8$ & 6033.3 & $14,650.3$ & $1,144,268.2$ \\
\hline 163 & & $291,899.5$ & $28,717.1$ & 5203.1 & $11,758.8$ & $1,124,629.8$ \\
\hline 164 & & $255,560.7$ & $27,485.2$ & 4982.8 & $13,246.9$ & $1,148,136.0$ \\
\hline 0.8 & \multirow{4}{*}{2002} & $266,058.3$ & $39,839.6$ & 7610.4 & $19,978.0$ & $1,164,239.0$ \\
\hline 166 & & $339,385.1$ & $40,055.4$ & 7384.7 & $20,390.1$ & $1,182,576.3$ \\
\hline 167 & & $390,466.3$ & $38,117.8$ & 6368.6 & $16,365.8$ & $1,181,000.0$ \\
\hline 168 & & $341,856.8$ & $36,482.7$ & 6099.0 & $18,437.0$ & $1,197,270.8$ \\
\hline 169 & \multirow{4}{*}{2003} & $606,640.0$ & $47,242.2$ & 9196.4 & $24,033.7$ & $1,625,546.2$ \\
\hline 170 & & $773,832.5$ & $47,498.1$ & 8923.7 & $24,529.5$ & $1,735,802.5$ \\
\hline 171 & & $890,302.8$ & $45,200.4$ & 7695.9 & $19,688.2$ & $1,792,349.4$ \\
\hline 172 & & $779,468.2$ & $43,261.5$ & 7370.0 & $22,179.8$ & $1,758,882.6$ \\
\hline
\end{tabular}


C. O. Arimie et al.

\section{Continued}

\begin{tabular}{|c|c|c|c|c|c|c|}
\hline 173 & \multirow{4}{*}{2004} & $651,425.4$ & $52,157.4$ & $11,201.4$ & $28,295.5$ & $2,039,515.9$ \\
\hline 174 & & $830,961.1$ & $15,439.9$ & $10,869.2$ & $28,878.9$ & $2,127,893.3$ \\
\hline 175 & & $956,029.9$ & $49,903.2$ & 9373.7 & $23,179.2$ & $2,171,579.1$ \\
\hline 176 & & $837,012.8$ & $47,762.8$ & 9876.8 & $26,112.6$ & $2,148,243.2$ \\
\hline 177 & \multirow{4}{*}{2005} & $618,328.3$ & $55,588.3$ & $11,560.0$ & $36,012.1$ & $2,831,255.6$ \\
\hline 178 & & $811,555.8$ & $59,516.5$ & $12,676.1$ & $38,494.5$ & $2,592,273.2$ \\
\hline 179 & & $1,062,751.7$ & $62,650.6$ & $13,211.3$ & $13,473.9$ & $2,985,541.8$ \\
\hline 180 & & $98,460.7$ & $66,137.2$ & $13,810.1$ & $42,106.0$ & $3,201,996.4$ \\
\hline 181 & \multirow{4}{*}{2006} & $749,944.0$ & $73,375.5$ & $15,212.4$ & $37,696.9$ & $3,169,613.4$ \\
\hline 182 & & $986,377.9$ & $77,684.6$ & $15,388.1$ & $40,821.5$ & $3,399,351.8$ \\
\hline 183 & & $1,292,413.0$ & $79,508.8$ & $15,504.6$ & $47,099.6$ & $3,924,775.0$ \\
\hline 184 & & $1,199,547.3$ & $82,688.3$ & $15,620.1$ & $44,360.0$ & $4,078,498.8$ \\
\hline 185 & \multirow{4}{*}{2007} & $931,325.0$ & $87,245.7$ & $19,471.8$ & $44,769.1$ & $3,986,279.5$ \\
\hline 186 & & $1,232,947.2$ & $89,714.4$ & $18,637.2$ & $46,705.8$ & $4,426,083.8$ \\
\hline 187 & & $1,619,235.4$ & $104,601.5$ & $17,928.5$ & $53,776.4$ & $4,986,489.4$ \\
\hline 188 & & $1,506,111.4$ & $97,341.0$ & $17,423.6$ & $61,203.9$ & 5165742.0 \\
\hline 189 & \multirow{4}{*}{2008} & $1,087,907.7$ & $104,146.3$ & $22,167.2$ & $49,557.1$ & $4,740,806.8$ \\
\hline 190 & & $1,440,102.2$ & $99,366.7$ & $21,231.4$ & $60,332.3$ & $4,853,839.3$ \\
\hline 191 & & $1,889,913.4$ & $118,850.9$ & $20,494.6$ & $58,139.3$ & $5,524,363.8$ \\
\hline 192 & & $1,606,557.7$ & $111,787.6$ & $19,918.7$ & $66,494.3$ & $5,538,294.6$ \\
\hline 193 & \multirow{4}{*}{2009} & $1,148,460.0$ & $111,750.0$ & $23,920.0$ & $54,710.0$ & $5,421,316.8$ \\
\hline 194 & & $1,554,120.9$ & $114,084.0$ & $24,401.1$ & $57,072.4$ & $5,704,400.3$ \\
\hline 195 & & $2,074,683.0$ & $135,850.8$ & $24,417.0$ & $64,352.7$ & $6,340,199.4$ \\
\hline 196 & & $1,767,306.1$ & $120,471.0$ & $21,534.9$ & $62,474.5$ & $6,376,225.2$ \\
\hline
\end{tabular}

